\title{
Karnaugh-Map Utility in Medical Studies: The Case of Fetal Malnutrition
}

\author{
Rufaidah Ali Rushdi \\ Department of Pediatrics \\ Kasr Al-Ainy School of Medicine, Cairo University \\ Cairo, 11562, Arab Republic of Egypt \\ Ali Muhammad Rushdi* \\ Department of Electrical and Computer Engineering \\ King Abdulaziz University, Jeddah 21589, Saudi Arabia \\ *Corresponding author: arushdi@kau.edu.sa
}

(Received November 9, 2017; Accepted January 3, 2018)

\begin{abstract}
This paper advocate and demonstrates the utility of the Karnaugh map, as a pictorial manual tool of Boolean algebra, in the exploration of medical problems as exemplified herein by the problem of Fetal Malnutrition $(F M)$. The paper briefly introduces the $F M$ problem, and specifies four metrics or tests used frequently in its study. Clinical data collected about these metrics (as continuous variables or dichotomized versions thereof) are conventionally treated via statistical methods. The Karnaugh map serves as a convenient way for aggregating the set of clinical data available into a pseudo-Boolean function. The map can be used to produce a two-by-two contingency matrix (confusion matrix or frequency matrix) that relates an assessed test or metric to a reference or standard one. Each of these two metrics can be any of the map variables or a function of some or all of these variables. While the map serves in this capacity as a supplement or aid to statistical methods, it is also useful for certain non-statistical methods (specifically Boolean ones). The paper shows how the map entries can be dichotomized via an appropriate threshold for use in Boolean Analysis $(B A)$, which can be conducted despite the lack of a gold standard. The map also implements Qualitative Comparative Analysis $(Q C A)$ for the given clinical data. The map variable-handling capability does not pose as a shortcoming for either $B A$ or $Q C A$, since the number of variables involved (not only herein but in other typical medical problems as well) is relatively small. The concepts and methods introduced herein are demonstrated through application to the aforementioned set of clinical data for the $F M$ problem, and can be extended to a wide variety of medical problems.
\end{abstract}

Keywords- Karnaugh map, Contingency table, Gold standard, Fetal malnutrition, Pseudo-Boolean function, Boolean analysis, Qualitative comparative analysis, Epidemiology, Diagnostic testing.

\section{Introduction}

The Karnaugh map is simply a special form of the truth table, which enjoys the bonus merits of two-dimensionality and visual adjacency, and is, therefore, more convenient for handling combinatorial problems arising in any field. In this paper, we explore the utility of the Karnaugh map in medical research both as an aid for manual calculation of real statistical quantities and as a tool for insightful interpretation of dichotomized or Boolean quantities. Our demonstrative running example uses data concerning four metrics or tests for the medical problem of Fetal Malnutrition $(F M)$, a very important problem in neonatology and pediatrics. These data have been recently collected for a cross-sectional population of full-term and preterm infants in Cairo, Egypt (Rushdi, 2017).

The fact that there is a widespread and extensive use of statistical methods in medical research (Zhou et al., 2009) attests to the power and usefulness of these methods in interpreting 
International Journal of Mathematical, Engineering and Management Sciences

Vol. 3, No. 3, 220-244, 2018

ISSN: 2455-7749

experimental data. However, certain qualifying remarks and observations should be added concerning the correctness, validity and nature of that use. Critical reviewers of the biomedical literature have consistently found that about half the articles that used statistical methods did so incorrectly (Glantz, 1980; Lang, 2004). Nowadays, statistical errors seem to remain common in the medical literature, even in the top journals of basic and clinical sciences, and hence closer attention to statistical methodology should be seriously considered so as to raise standards (Strasak et al., 2007). Despite its effectiveness in handling genuine data, statistics are of limited power in detecting (the not uncommon) false or fabricated data, although a few statistical tools do achieve this purpose, such as the Benford's law (De Felice et al., 2016). Gigerenzer and Marewski (2015) question the role of statistical inference as a universal method of science. They assert that this method, "advocated as the only game in town, is practiced in a compulsive, mechanical way - without judging whether it makes sense or not." Therefore, they conclude that "good science requires both statistical tools and informed judgment about what model to construct, what hypotheses to test, and what tools to use." We argue that informed judgment might be achieved or enhanced via qualitative but less elaborate (and hopefully less error-prone) methods such as Boolean-algebraic ones.

Boolean-algebraic techniques have been used in medicine for several decades (Lusted and Ledley, 1960; Feinstein, 1963; Marshall, 1986; 2001; Hammer and Bonates, 2006; Ogihara et al., 2013), However, the Karnaugh map, a major Boolean-algebraic tool, is virtually unheard of in medical circles. There are only very few medical references utilizing this map (see, e.g., Lin and Khatri, 2014; Van Loo and Romeijn, 2015; Bhambu and Kumar, 2015). We argue that the Karnaugh map has a good potential for more diverse and effective use in medical studies. This map has many definite advantages for pre-processing of quantitative data for statistical purposes. It is naturally suited for handling dichotomized or Boolean data and implementing qualitative techniques such as Boolean Analysis $(B A)$ and Qualitative Comparative Analysis $(Q C A)$. The map allows its user to reach minimal theories immediately without wasting effort and time pondering its constituent non-minimal ones. In map jargon, the map constructs prime-implicant loops without dwelling much on its constituent non-prime ones.

Since this paper is of interdisciplinary nature, a word of caution is needed to reconcile conflicting notations in the different fields involved. In standard medical practice, the term "positive" refers to the presence of an adverse condition such as a disease, while the opposite term "negative" refers to the absence of such a condition. By contrast, in many other walks of life, these two terms are suggestive of opposite meanings, as they are almost synonymous with "good" and "bad". Therefore, we deliberately (albeit arbitrarily) choose our logical (2-valued Boolean) variables such that an uncomplemented literal $(A)$ indicates absence of an adverse condition (the good phenomenon), while a complemented literal $(\bar{A})$ denotes its presence (the bad thing). Mathematically, we map the set $\{+1,-1\}$ used in medical jargon to the set $\{0,1\}$ (rather than the set $\{1,0\})$ used in logic terminology.

The Organization of the rest of this paper is as follows. Section 2 is a brief overview of the aforementioned recently-conducted study of the medical problem of Fetal Malnutrition. Section 3 digresses a little bit to explore the concept, ramifications and possible remedies for the lack of a perfect measure or gold standard of the studied problem. In Sec. 4, the Karnaugh map is used to represent the major findings of the aforementioned study as a pseudo-Boolean function. Section 5 shows how the Karnaugh map can be reduced to various contingency tables, which in turn are useful for producing many useful statistical measures or indicators. Sections 6 and 7 introduce the 
qualitative techniques of Boolean Analysis $(B A)$ and Qualitative Comparative Analysis ( $Q C A)$ from map perspective and demonstrate how and why the Karnaugh map can be used to implement these two techniques for the studied problem. Section 8 concludes this paper. To make the paper self-contained, an appendix is included to describe how a contingency table is used to derive the measures or indicators typically employed in all types of diagnostic testing, in general, and in clinical or epidemiological testing, in particular.

\section{Description of the Medical Problem 2.1 Fetal Malnutrition}

Fetal Malnutrition $(F M)$ is an adverse clinical condition resulting from the failure of the fetus to acquire adequate amounts of subcutaneous fat and muscle mass during its intrauterine life, with short and long term implications. An infant who is classified as Small for Gestational Age (SGA) may or may not have Fetal Malnutrition. The $S G A$ state is not synonymous (albeit associated) with $F M$. The importance of studying the $F M$ problem stems from the fact that $F M$ is associated with a higher risk of morbidity and mortality in the short and long terms (Rushdi, 2017).

\subsection{Metrics for FM Assessment}

Rushdi (2017) discusses several assessment methods (referred to herein as metrics) that have been proposed for assessing the presence or absence of $F M$. Initially, weight at birth was used as a common indicator of $F M$, but this practice was abandoned when $F M$ was found to occur at almost any birth weight. Later, an anthropometric assessment tool including several measurements was used to give what was thought to be a comprehensive evaluation of $F M$. The measurements covered body weight, length and head circumference, but it was soon discovered that these physical attributes may or may not be affected by $F M$. Finally, more appropriate proportionality indices were proposed. These include the Body Mass Index $(B M I)$, the Ponderal Index $(P I)$, and the Mid-Upper-Arm Circumference to Head Circumference ratio $(M U A C / H C)$. An alternative competitive index was developed based on the argument that neonatal morbidity and mortality are more closely related to the nutritional status of a newborn at birth than to the birth weight for the gestational age. This index is called the Clinical Assessment of Nutritional Status (CANS) score. Without the use of any sophisticated equipment, the CANS score differentiates malnourished from appropriately nourished babies through systematized inspection and estimation of nine physical signs of nutrition including 1. Quality of hair, 2. Buccal fat in the cheeks, 3. Chin and neck fatness; loose wrinkled skin with the absence of subcutaneous fat in: 4 . Arms, 5. Back, 6. Buttocks, 7. Legs, 8. Chest, and 9. Abdomen. Each of these signs is rated from 1 (worst, severe $F M$ ) to 4 (best, well nourished). Therefore, the highest and lowest total CANS scores are 36 and 9, respectively. A total score of $<25$ is considered as $F M$. While the three anthropometric and proportionality indices $(B M I, P I, M U A C / H C)$ measure different aspects of the well-being of a newborn, the CANS score measures the visible wasting observed in malnourished newborns. All the four metrics, in one way or another, reflect the adverse intrauterine nutrition that FM newborns suffer. These four metrics were studied extensively by Rushdi (2017) via purely statistical techniques, and are studied herein via a mixture of statistical and combinatorial techniques. Table 1 lists pertinent information about these metrics, and uses upper-case variables to denote Boolean dichotomization of raw data expressed as lower-case variables. In particular, Table 1 shows that the threshold or cutoff values used in this dichotomization to indicate $F M$ presence are: CANS score $<25, B M I<11.2 \mathrm{~kg} / \mathrm{m}^{2}, P I<2.2 \mathrm{~g} / \mathrm{cm}^{3}$ and $M U A C / H C<0.27$. 
International Journal of Mathematical, Engineering and Management Sciences

Vol. 3, No. 3, 220-244, 2018

ISSN: 2455-7749

Table 1. The four metrics used for $F M$ assessment in Rushdi (2017) and in the current study

\begin{tabular}{|c|c|c|c|c|c|}
\hline $\begin{array}{l}\text { Metric Full and } \\
\text { Abbreviated Names }\end{array}$ & $\begin{array}{l}\text { Notation } \\
\text { (Raw } \\
\text { Variable) }\end{array}$ & $\begin{array}{c}\text { Nature of Raw } \\
\text { Variable }\end{array}$ & $\begin{array}{l}\text { Notation } \\
\text { (Boolean } \\
\text { Variable) } \\
\end{array}$ & $\begin{array}{c}\text { Condition for } F M \\
\text { Presence }(F M \\
\text { Positive }) \\
\end{array}$ & $\begin{array}{c}\text { Condition for } F M \\
\text { Absence (FM } \\
\text { Negative) } \\
\end{array}$ \\
\hline $\begin{array}{c}\text { Clinical Assessment of } \\
\text { Nutritional Status score } \\
\text { (CANS score) }\end{array}$ & 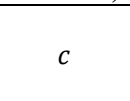 & $\begin{array}{l}\text { Integer-valued } \\
\text { dimensionless }\end{array}$ & 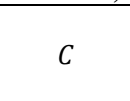 & $\begin{array}{c}c<25 \\
(C=0)\end{array}$ & $\begin{array}{c}c \geq 25 \\
(C=1)\end{array}$ \\
\hline Body Mass Index (BMI) & $b$ & $\begin{array}{c}\text { Real-valued of } \\
\text { dimension } \\
\text { mass/area }\end{array}$ & $B$ & $\begin{array}{c}b<11.2 \mathrm{~kg} / \mathrm{m}^{2} \\
\quad(B=0)\end{array}$ & $\begin{array}{c}b \geq 11.2 \mathrm{~kg} / \mathrm{m}^{2} \\
\quad(B=1)\end{array}$ \\
\hline Ponderal Index $(P I)$ & $p$ & $\begin{array}{l}\text { Real-valued of } \\
\text { dimension } \\
\text { mass/volume }\end{array}$ & $P$ & $\begin{array}{c}p<2.2 \mathrm{~g} / \mathrm{cm}^{3} \\
\quad(P=0)\end{array}$ & $\begin{array}{c}p \geq 2.2 \mathrm{~g} / \mathrm{cm}^{3} \\
(P=1)\end{array}$ \\
\hline $\begin{array}{c}\text { Mid-Upper-Arm } \\
\text { Circumference to Head } \\
\text { Circumference ratio } \\
(M U A C / H C)\end{array}$ & $m$ & $\begin{array}{l}\text { Real-valued } \\
\text { dimensionless }\end{array}$ & $M$ & $\begin{array}{c}m<0.27 \\
(M=0)\end{array}$ & $\begin{array}{c}m \geq 0.27 \\
(M=1)\end{array}$ \\
\hline
\end{tabular}

\subsection{Data Collection}

The data used in the current work was collected by Rushdi (2017) as part of her M.Sc. thesis work. A multi-center cross-sectional sample of full-term and preterm infants was studied between November 2016 and October $2017(N=301)$. These infants were all born at some Teaching Hospitals in Cairo, Egypt (Kasr Al-Ainy Hospitals of Cairo University or Al-Galaa Hospital). Early neonatal morbidities were detected within the first week of life for a subgroup of 50 infants within this sample, who required admission to the Neonatal Intensive Care Unit $(N I C U)$. The CANS score was evaluated for all infants enrolled in the study within 48 hours of birth. Moreover, measurements and calculations were performed for the Body Mass Index (BMI), the Ponderal Index $(P I)$, and the Mid-Upper-Arm Circumference/Head Circumference ratio $(M U A C / H C)$. Other data were also collected and reported in Rushdi (2017). However, all we need in the present study is the raw data of the four assessment metrics, with a stress on its dichotomization into Boolean data.

\section{Lack of a Gold Standard}

A gold standard test is the best single test (or a combination of tests) that is considered the currently preferred method of diagnosing a particular disease $(X)$. All other methods of diagnosing the disease $X$, including any new test, need to be compared against this 'gold' standard (Parikh et al., 2008). Whenever a gold standard is available, it is usually invasive or expensive. There is no agreed-upon gold standard among diagnostic tests for many medical problems, including the present case study of Fetal Malnutrition $(F M)$. This fact is one motivation of a hot area of ongoing research in scientific methodology, dealing with the evaluation of diagnostic tests in the absence of a gold standard (Joseph et al., 1995; Reitsma et al., 2009; Dendukuri et al., 2012). The outcome of this research is still far from being conclusive. Although many methods have been explored, none of them was found to be reasonably satisfactory. There is a unanimous agreement among prominent researchers dealing with this problem that it is "an area where we are still awaiting a solution." In fact, the most prominent proposed technique, viz., Discrepant Analysis has already been completely discredited. Besides, this analysis necessitates replication of measurements or testing in disputed cases, something extremely difficult in many studies, and next to impossible in our present study. There are techniques that are more realistic such as Latent Class Analysis (Rindskopf and Rindskopf, 1986), but they have scale-up problems due to mathematical complexity and unavailability of convenient software. More promising techniques 
International Journal of Mathematical, Engineering and Management Sciences

Vol. 3, No. 3, 220-244, 2018

ISSN: 2455-7749

that are easy to use, suffer from poorly-understood inherent biases. The best candidate among these techniques is to use a Composite Reference Standard (CRS) (Alonzo and Pepe, 1999). This composite standard is obtained through voting among the competing standards, i.e., using the weighted sum of the competing standards, with weights decided by expert opinions. Of course, these opinions are subject to uncontrolled variability and liable to being unreasonably biased. Since there is no globally accepted gold standard for assessing $F M$ after birth, Rushdi (2017) adopts the tentative solution of alternating between various $F M$ metrics such that each of them is arbitrarily given a chance to act as the gold standard against which to evaluate the other $F M$ clinical tests.

\section{Representation via a Pseudo-Boolean Function}

A pseudo-switching (or pseudo-Boolean) function $N: B_{2}^{n} \rightarrow R$ is a function whose domain is similar to that of a switching function $g: B_{2}^{n} \rightarrow B_{2}$, but whose range is the real line (or a subset thereof), by contrast to the range of $g$ which is the switching or two-valued Boolean set $B_{2}=$ $\{0,1\}$ (Rushdi and Ba-Rukab, 2017). Therefore, the Karnaugh map for $N$ has the same structure or input variables as $g$ has, but its entries (values of the output variable) are reals that are not necessarily restricted to the values 0 and 1. The 301 cases studied in Sec. 2 are distributed among the 16 possible configurations or combinations (truth-table lines or Karnaugh-map cells) set by the binary variables $C, B, P$ and $M$. Such a distribution is given by the following pseudoswitching function $N(C, B, P, M)$ expressed in its minterm expansion

$N(C, B, P, M)=26 \bar{C} \bar{B} \bar{P} \bar{M}+16 \bar{C} \bar{B} \bar{P} M+6 \bar{C} \bar{B} P \bar{M}+8 \bar{C} \bar{B} P M+(0) \bar{C} B \bar{P} \bar{M}+(0) \bar{C} B \bar{P} M+$ $4 \bar{C} B P \bar{M}+30 \bar{C} B P M+$ (1) $C \bar{B} \bar{P} \bar{M}+(6) C \bar{B} \bar{P} M+$ (1) $C \bar{B} P \bar{M}+$ (6) $C \bar{B} P M+$ (0) $C B \bar{P} \bar{M}+$ (1) $C B \bar{P} M+(4) C B P \bar{M}+(192) C B P M$

We stress that the symbol " + " used in (1) denotes arithmetic addition (as usual!), and does not mean logical OR operation (for which we employ the specific symbol " $\vee$ " herein). Here, $N: B_{2}^{4} \rightarrow Z$, where $Z$ is the set of non-negative integers. The (parenthetic) discriminant associated with a certain minterm is a non-negative integer indicating the number of cases reported in Rushdi (2017) for the configuration of this minterm. For example, the integer 8 associated with the minterm $\bar{C} \bar{B} P M$ means that in 8 of the cases both the CANS score and the BMI metric indicated presence of the Fetal Malnutrition condition, while both the $P I$ metric and the $M U A C / H C$ indicated the absence of that condition. The sum of all discriminants is the total number of cases (301), as anticipated.

A notable advantage of the Karnaugh map is that it can be conveniently used to represent any pseudo-switching function. Fig 1 shows a Karnaugh-map representation of the pseudo-switching function $N(C, B, P, M)$ given in (1). The discriminants associated with various minterms now appear as entries of the corresponding map cells. Though the true prevalence of Fetal Malnutrition is unknown, the map indicates quickly that the four metrics used suggest that its value in not really high. There is a clear pattern of a pile up of individual cases at or near two particular faraway cells of the map. In fact, the largest map entry (192) appears in the all-1 cell (the CBPM cell), which means that the four metrics unanimously agree that the aforementioned disease condition is absent in 192 cases. The third largest map entry (26) appears in the all-0 cell (the $\bar{C} \bar{B} \bar{P} \bar{M}$ cell), which means that the four metrics unanimously agree that this disease condition is present in 26 cases. If we ignore other map entries, we may roughly estimate disease prevalence to be $(26 /(26+192))$ which corresponds to 0.119 or $11.9 \%$. The four metrics vote $3: 1$ 
International Journal of Mathematical, Engineering and Management Sciences

Vol. 3, No. 3, 220-244, 2018

ISSN: 2455-7749

for disease absence in 41 cases, obtained as the sum of entries in the four cells adjacent to the all1 cell (the four cells $\bar{C} B P M, C \bar{B} P M, C B \bar{P} M, C B P \bar{M}$ ), and vote 3:1 for disease presence in 23 cases, obtained as the sum of entries in the four cells adjacent to the all- 0 cell (the four cells $\bar{C} \bar{B} \bar{P} M, \quad \bar{C} \bar{B} P \bar{M}, \bar{C} B \bar{P} \bar{M}$, and $C \bar{B} \bar{P} \bar{M})$. With these numbers, we might update our estimate of disease prevalence to become $((26+23) /(26+23+41+192))$ which corresponds to 0.174 or 17.4 $\%$. The four metrics have a tie (vote $2: 2)$ in 19 cases $(6.3 \%$ of all case) distributed among the six cells $\bar{C} \bar{B} P M, \bar{C} B \bar{P} M, \bar{C} B P \bar{M}, C \bar{B} \bar{P} M, C \bar{B} P \bar{M}$, and $C B \bar{P} \bar{M}$.

Before closing this section, we remark that it is possible to normalize the entries of the map in Fig. 1 by dividing each of them by their sum of 301. In this case the map becomes a crossclassification table and its entries might be interpreted as probabilities, which could be studied in terms of the multinomial distribution (O’Neill, 2015).

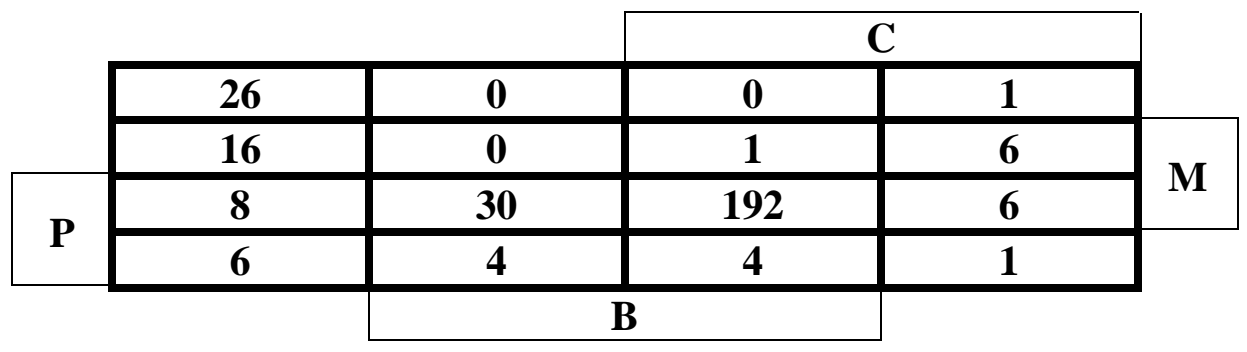

$N(C, B, P, M)$

Fig. 1. Karnaugh-map representation of the Pseudo-Boolean function $N(C, B, P, M)$ representing the numbers of cases observed by Rushdi (2017) in various configurations

\section{Reduction to Two-by-Two Contingency Matrices}

To convert the function $N(C, B, P, M)$ to a function representing a contingency matrix (also named a confusion matrix or a frequency matrix), we introduce the concept of arithmetic join derivative or additive eliminant (in analogy to the concept of disjunctive eliminant of a Boolean function (Brown, 1990)). For example, the additive eliminant of $N(C, B, P, M)$ w.r.t. $B, P$, and $M$ is given by

$$
\begin{gathered}
\begin{array}{c}
\alpha^{3} N / \alpha B \alpha P \alpha M=N(C, 0,0,0)+N(C, 0,0,1)+N(C, 0,1,0)+N(C, 0,1,1)+ \\
N(C, 1,0,0)+N(C, 1,0,1)+N(C, 1,1,0)+N(C, 1,1,1) \\
=(26+16+6+8+0+0+4+30) \bar{C}+(1+6+1+6+0+1+4+192) C \\
=90 \bar{C}+211 C=90 \bar{C} \bar{C}+(0) \bar{C} C+(0) C \bar{C}+211 C C
\end{array}
\end{gathered}
$$

The additive eliminant of $N(C, B, P, M)$ with respect to $P$ and $M$ is given by

$$
\begin{aligned}
& \alpha^{2} N / \alpha P \alpha M=N(C, B, 0,0)+N(C, B, 0,1)+N(C, B, 1,0)+N(C, B, 1,1)= \\
& (26+16+6+8) \bar{C} \bar{B}+(0+0+4+30) \bar{C} B+(1+6+1+6) C \bar{B}+(0+1+4+192) C B \\
& =56 \bar{C} \bar{B}+34 \bar{C} B+14 C \bar{B}+197 C B
\end{aligned}
$$

Obviously, the additive eliminant of $N(C, B, P, M)$ with respect to all four arguments is the sum of all discriminants, i.e., 301. 
International Journal of Mathematical, Engineering and Management Sciences

Vol. 3, No. 3, 220-244, 2018

ISSN: 2455-7749

The Karnaugh map is conveniently suitable for implementing additive elimination as a result for its capability to handle "differentiation" on Boolean domains (Rushdi, 1986). Fig. 2 reduces the 4variable Karnaugh map in its left-hand side to the 1-variable map in its right-hand side through the additive elimination of $N(C, B, P, M)$ with respect to $B, P$, and $M$ (visually implementing equation (2)). The resulting map is drawn as though it is a 2-variable map of the same variable $C$ so as to represent the contingency table of $C$ with respect to itself, which is (trivially!) a case of perfect positive association. In essentially the same fashion, Fig. 3 reduces the 4-variable Karnaugh map in its left-hand side to the 2-variable map in its right-hand side via additive elimination of $N(C, B, P, M)$ with respect to $P$ and $M$ (visually implementing equation (3)). The resulting map represents the contingency table of $C$ with respect to $B$. Similarly, Figs. 4 and 5 obtain the contingency tables of $C$ with respect to $P$, and of $C$ with respect to $M$, respectively. Fig. 6 deals with map construction of the contingency table of $C$ with respect to $V$, where $V$ is the majority voting or the 2-out-of-3 function of the three anthropometry variables $B, P$, and $M$. Its uncomplemented and complemented versions are given by

$$
\begin{gathered}
V=B P \vee P M \vee B M=B P M \vee B P \bar{M} \vee B \bar{P} M \vee \bar{B} P M \\
\bar{V}=\bar{B} \bar{P} \vee \bar{P} \bar{M} \vee \bar{B} \bar{M}=\bar{B} \bar{P} \bar{M} \vee \bar{B} \bar{P} M \vee B \bar{P} \bar{M} \vee \bar{B} P \bar{M}
\end{gathered}
$$

Fig. 6 is an example where one of the variables of the resulting contingency matrix is not a variable of the original Karnaugh map per se, but rather a function of several variables of that map. Fig. 7 differs from its predecessors, since it is not produced out of the map in Fig. 1, and (for comparison purposes) it deliberately violates the requirement that an assessed metric should not be a part of the information used to construct the reference metric. In fact, Fig. 7 displays the contingency table of $C$ with respect to $F$, where $F$ is an expert-independent $C R S$ given by the overall real voting function, i.e., $F=0$ when $f<1$ and $F=1$ when $f \geq 1$, and $f$ is the average of the weighted raw metrics, wherein each metric is normalized by its own threshold or cutoff, i.e., $f$ is given by

$f=(c / 25+b / 11.2+p / 2.2+m / 0.27) / 4$

The Boolean variable $F$ is defined to indicate the absence of Fetal Malnutrition when all four metrics unanimously agree it is absent. It also asserts $F M$ presence when all four metrics attest to this presence. The proposed scheme performs dichotomization after voting, thereby avoiding the loss of information that might result from dichotomization before voting. In fact, the scheme considers a metric more decisive (and consequently deserving of more weight) the farthest it is from its threshold. As a bonus, this scheme avoids the ambiguity that faces Boolean voting when a tie takes place.

Once a two-by-two contingency table is obtained, many useful measures or indicators can be evaluated (see Appendix A). Table 2 presents a small sample of such measures or indicators. Each of the indicators in this sample is a probability, and might have been multiplied by 100 to denote a percentage. 
International Journal of Mathematical, Engineering and Management Sciences

Vol. 3, No. 3, 220-244, 2018

ISSN: 2455-7749
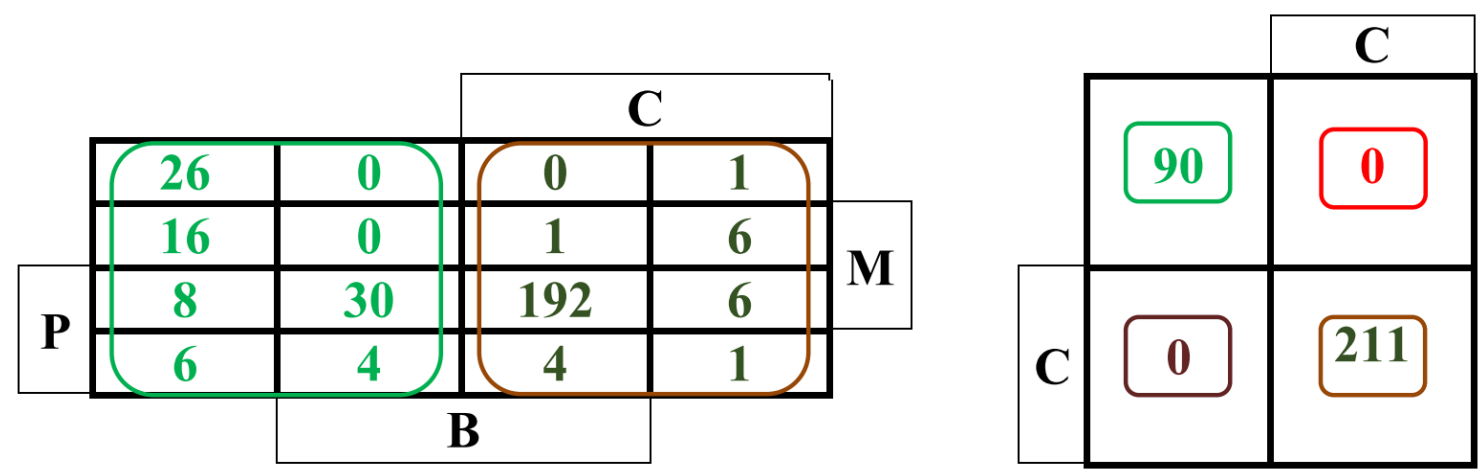

Fig. 2. Map additive elimination of $N(C, B, P, M)$ with respect to $B, P$, and $M$ to obtain the contingency table of $C$ with respect to itself
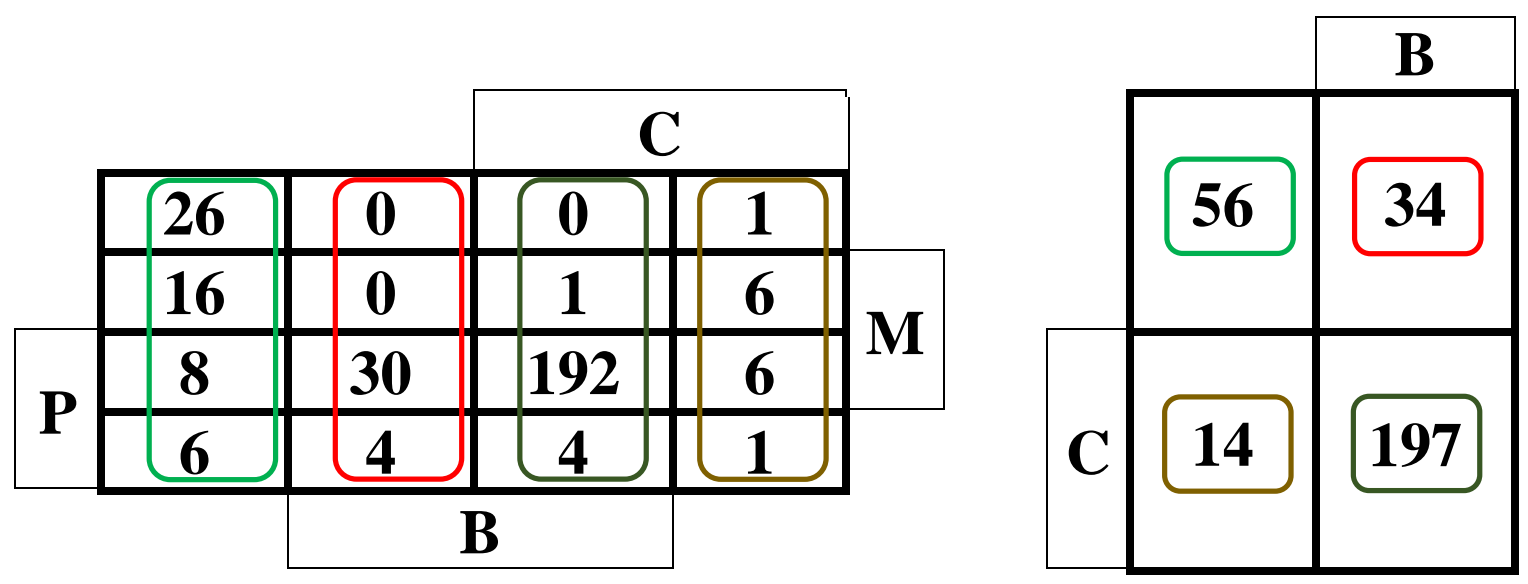

Fig. 3. Map additive elimination of $N(C, B, P, M)$ with respect to $P$ and $M$ to obtain the contingency table of $C$ with respect to $B$
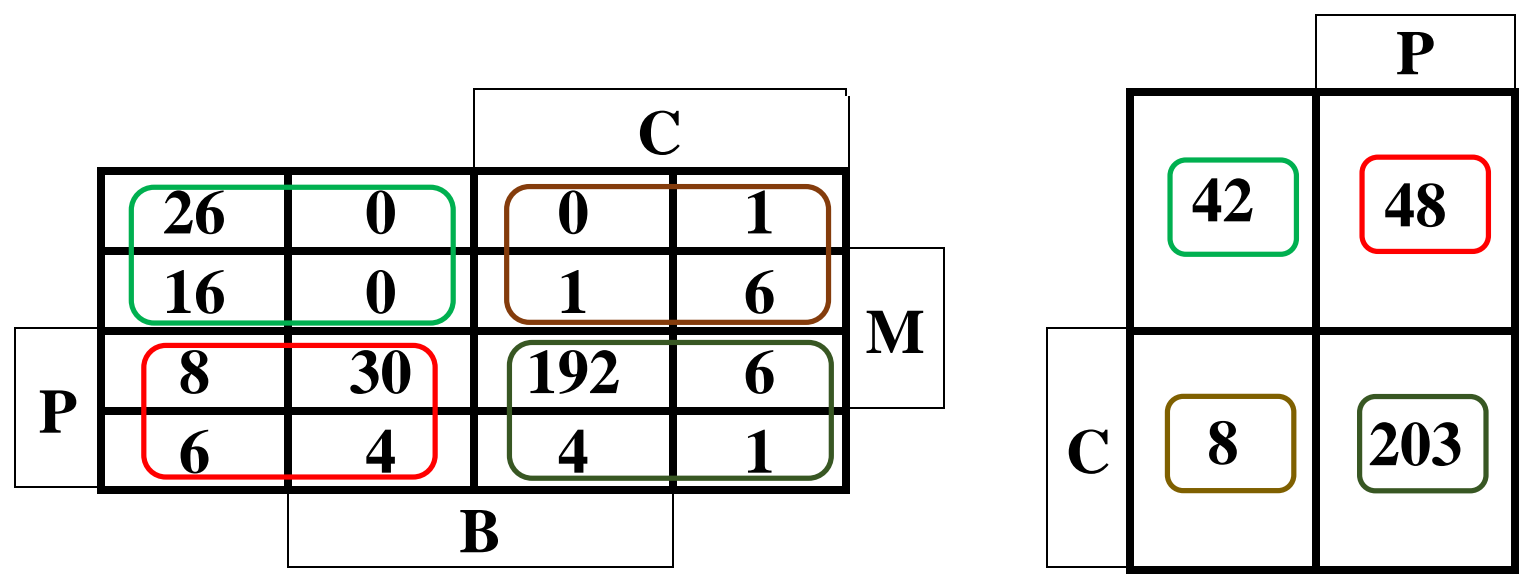

Fig. 4. Map additive elimination of $N(C, B, P, M)$ with respect to $B$ and $M$ to obtain the contingency table of $C$ with respect to $P$ 
International Journal of Mathematical, Engineering and Management Sciences

Vol. 3, No. 3, 220-244, 2018

ISSN: 2455-7749
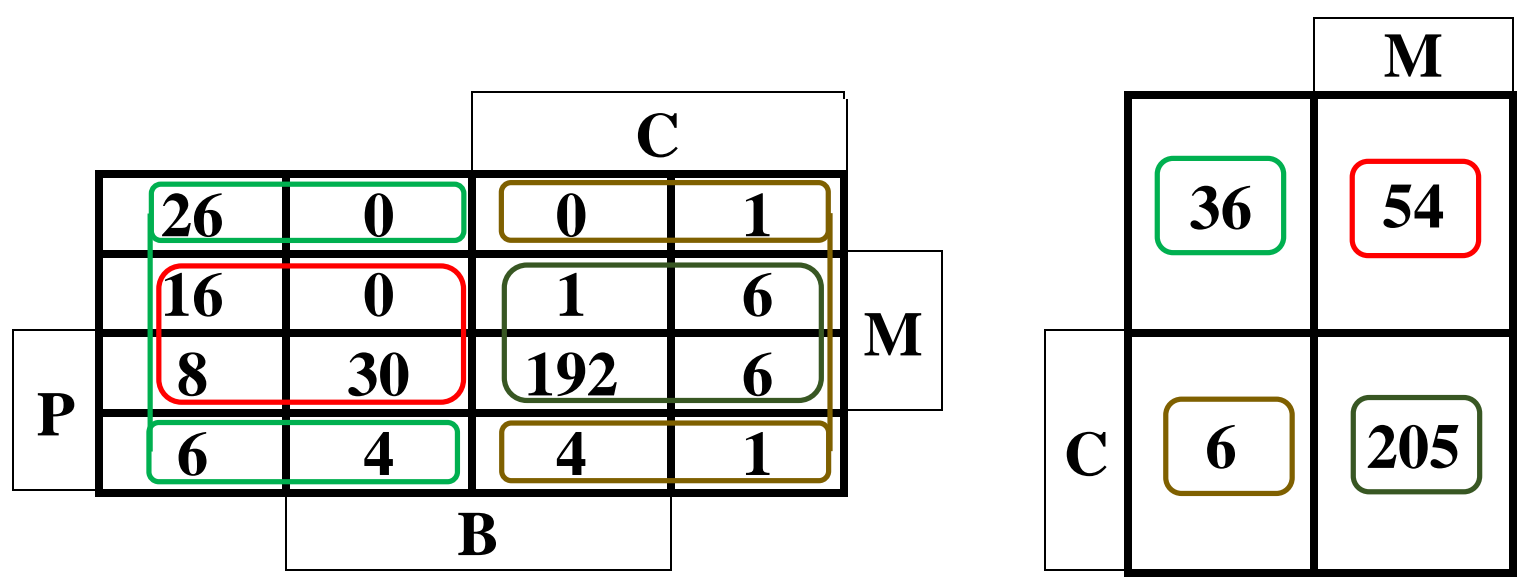

Fig. 5. Map additive elimination of $N(C, B, P, M)$ with respect to $B$ and $P$ to obtain the contingency table of $C$ with respect to $M$
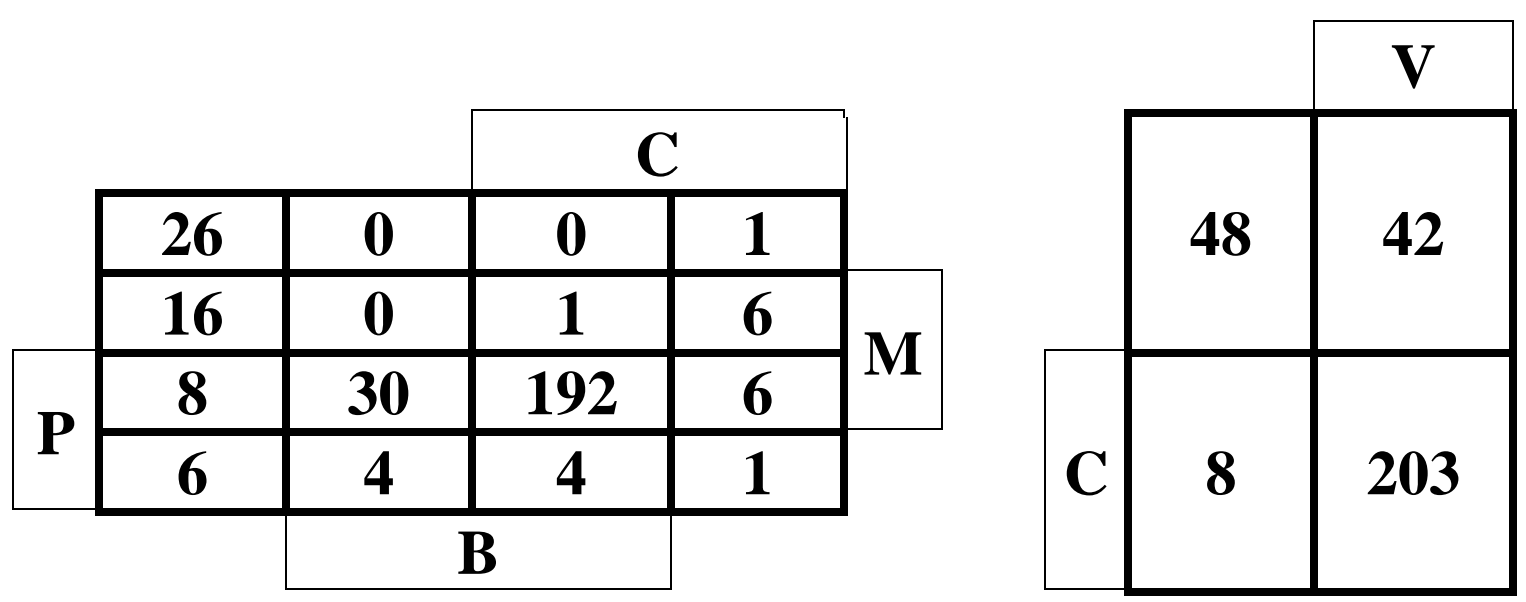

Fig. 6. Map construction of the contingency table of $C$ with respect to $V$, where $V$ is the voting function (the 2-out-of-3 function) $B P \vee P M \vee M B$

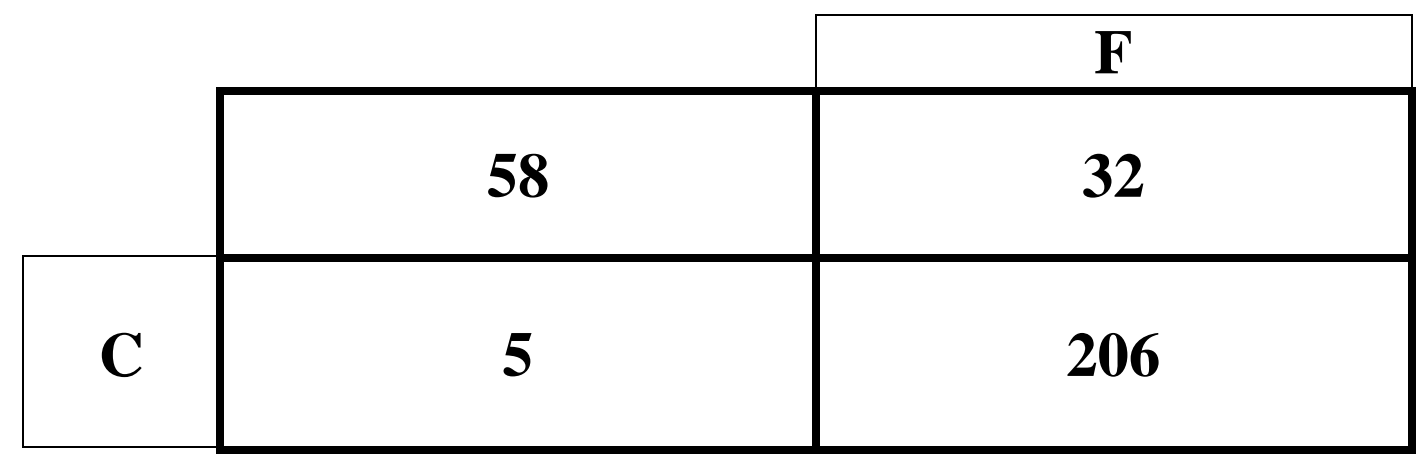

Fig. 7. The contingency table of $C$ with respect to $F$, where $F$ is the overall real voting function 
International Journal of Mathematical, Engineering and Management Sciences

Vol. 3, No. 3, 220-244, 2018

ISSN: 2455-7749

Table 2. Various direct and reverse measures of the $C A N S$ score $(C)$ with respect to different metrics (including $C$ itself), wherein each measure might be multiplied by 100 to express a percentage

\begin{tabular}{|c|c|c|c|c|c|c|c|}
\hline Metric & Sensitivity & Specificity & $P P V$ & $N P V$ & $\begin{array}{c}\text { Error of the First } \\
\text { Kind }\end{array}$ & $\begin{array}{c}\text { Error of the } \\
\text { Second Kind }\end{array}$ & $\begin{array}{c}\text { Total } \\
\text { Error }\end{array}$ \\
\hline$C A N S$ score $(C)$ & 1.00 & 1.00 & 1.00 & 1.00 & 0 & 0 & 0 \\
\hline$B M I(B)$ & 0.80 & 0.8528 & 0.6222 & 0.9336 & 0.1130 & 0.0465 & 0.1595 \\
\hline$P I(P)$ & 0.84 & 0.8088 & 0.4667 & 0.9621 & 0.1595 & 0.0266 & 0.1860 \\
\hline$M U A C / H C(M)$ & 0.8571 & 0.7915 & 0.4000 & 0.9716 & 0.1794 & 0.0199 & 0.1993 \\
\hline $\begin{array}{c}\text { Anthropometric Majority } \\
\text { Voting }(V)\end{array}$ & 0.8571 & 0.8286 & 0.5333 & 0.9621 & 0.1395 & 0.0266 & 0.1661 \\
\hline Overall Real Voting $(F)$ & 0.9207 & 0.8655 & 0.6444 & 0.9763 & 0.1063 & 0.0166 & 0.1229 \\
\hline
\end{tabular}

\section{Boolean Analysis}

Boolean Analysis $(B A)$ was introduced by Flament $(1965,1976)$ for application to questionnaire data (Degenne and Lebeaux, 1996). This analysis is a partial-order generalization of scalogram analysis, and is particularly useful for hierarchical data (Theuns, 1999), and generally any type of data that can be dichotomized, i.e., reduced to optimal binary data. Essentially, $B A$ is based on the determination of an ideal for a Boolean algebra and of its quotient algebra (Theuns, 1989). A notable offshoot of $B A$ is Coincidence Analysis $(C A N)$, which is used in causal reasoning that uncovers deterministic causal structures (Baumgartner, 2009; Rushdi and Badawi, 2017b).

This section utilizes the Karnaugh map as a tool of Boolean Analysis for exploring Boolean (noncausal) relations among the four measures $(C, B, P$, and $M)$ of Fetal Malnutrition used herein. Such an exploration does not require the availability of a perfect metric or gold standard. Though our analysis uses the common mathematics of $B A$ and $C A N$, it follows the usual interpretations of mainstream $B A$ (rather than those of $C A N$ ), which means that the implications discovered have no causal connotations.

The Karnaugh map is used to represent a two-valued Boolean function $g_{T}(C, B, P, M)$, which is 1 for definitely observed (instantiated) cells or configurations, and which is 0 for absent (or couldbe-ignored) ones. In the basic method of $B A$, a Boolean function $g_{0}(C, B, P, M)$ is derived from the pseudo-Boolean function $N(C, B, P, M)$, such that

$$
\begin{aligned}
& g_{0}(C, B, P, M)=0, \text { if } N(C, B, P, M)=0 \\
& g_{0}(C, B, P, M)=1, \text { if } N(C, B, P, M)>0
\end{aligned}
$$

In an advanced version of $B A$ (Theuns, 1989; 1994), dichotomization of the pertinent population $N(C, B, P, M)$ is achieved such that a "small" part of the population is ignored, resulting in better (more compact or more plausible) conclusions or more intelligible implicational graphs. Specifically, Equations (6) are generalized to derive a Boolean function $g_{T}(C, B, P, M)$, based on a certain non-negative threshold $T$ such that

$$
\begin{aligned}
& g_{T}(C, B, P, M)=0, \text { if } N(C, B, P, M) \leq T \\
& g_{T}(C, B, P, M)=1, \text { if } N(C, B, P, M)>T
\end{aligned}
$$


International Journal of Mathematical, Engineering and Management Sciences

Vol. 3, No. 3, 220-244, 2018

ISSN: 2455-7749

To ensure that indeed only a "small" part of the population is ignored, the threshold $T$ is chosen to be an integer that is strictly less than half the average number of cases per configuration, i.e. $(T<$ $(301 / 16) / 2)$ or $(T \leq 9)$. The only possible values of $T$ yielding distinct $g_{T}$ are $0,1,4,6$, and 8 . The resulting functions satisfy the order relations $\left(\bar{g}_{0}<\bar{g}_{1}<\bar{g}_{4}<\bar{g}_{6}<\bar{g}_{8}\right)$, and are displayed by the Karnaugh maps in Figs. 8(a)-8(e). In passing, we note that dichotomization of continuous variables is generally not welcome for conventional statistical data analysis (Royston et al., 2006; DeCoster et al., 2009), but in BA, dichotomization is the essence of the method as well as the source of its great simplicity.

We are now interested in obtaining the complete sum (CS) (Blake Canonical Form) (Brown, 1990; Rushdi and Badawi, 2017b; Rushdi and Rushdi, 2018) of the complement $\bar{g}_{T}$ of the dichotomized function $g_{T}$, which is the disjunction of all the prime implicants (PIs) of $\bar{g}_{T}$. Flament (1976) called these prime implicants "Projections Canoniques Ultimes" (the French for Ultimate Canonical Projections), abbreviated to PCUs (Theuns, 1989). When the prime implicants are equated to zero, they are called prime consequents in Boolean reasoning (Brown, 1990) and minimal theories in Coincidence Analysis (Baumgartner, 2009; Rushdi and Badawi, $2017 b)$. For the present small number of variables, the Karnaugh map allows obtaining $\operatorname{CS}\left(\bar{g}_{T}\right)$ and hence all prime consequents or minimal theories, thanks to the adjacency property of the map cells.

For example, the Karnaugh map in Fig. 8(a) allows drawing the two prime-implicant loops for $\bar{g}_{0}$ and hence obtaining the required complete sum as

$\operatorname{CS}\left(\bar{g}_{0}\right)=B \bar{P} \bar{M} \vee \bar{C} B \bar{P}=0$

An important consequence of (8) is that each of the terms in it is individually equal to 0 (Brown, 1990, Rushdi and Rushdi, 2018), thereby representing two minimal theories, namely:

$B \bar{P} \bar{M}=0$

$\bar{C} B \bar{P}=0$

Similarly, we show all prime-implicant loops for the function $\bar{g}_{1}$ in Fig. 8(b). We also show the prime-implicant loops for the functions $\bar{g}_{4}, \bar{g}_{6}$ and $\bar{g}_{8}$ in the Karnaugh maps in Figs. 8(c)-8(e), wherein each succeeding function inherits or shares PIs with preceding functions. All possible minimal theories for various threshold values are obtained and listed in Table 3. All theories obtained involve two or three literals only, and hence meet the simplicity criterion set by Theuns (1989). Therefore, we refrain from giving preference to a specific set of theories based on a certain threshold. Such preference is left to the discretion of a user of our results, and depends on how large the threshold acceptable by this user could be.

In passing, we note the fact that an n-literal minimal theory in equational form is equivalent to any of $2^{n}$ clausal or implicational forms, where $n \geq 1$ (Brown, 1990; Rushdi and Badawi, 2017b). We demonstrate this fact in Table 4 by displaying the four implicational forms equivalent to the 2-literal minimal theory $(B \bar{P}=0)$, and the eight implicational forms that are equivalent to the 3 literal minimal theory $(B \bar{P} \bar{M}=0)$. The four clausal interpretations of the minimal theory $(B \bar{P}=$ 
International Journal of Mathematical, Engineering and Management Sciences

Vol. 3, No. 3, 220-244, 2018

ISSN: 2455-7749

0 ) (which is valid for $T=1$, subject to ignoring just a single reported case) can be stated verbally as

(i) It is not the case that $B M I$ indicates absence of Fetal Malnutrition and $P I$ indicates its presence $(B \bar{P} \rightarrow 0)$,

(ii) If $B M I$ asserts absence of Fetal Malnutrition, then $P I$ does also so $(B \rightarrow P)$,

(iii) If $P I$ asserts presence of Fetal Malnutrition, then $B M I$ does also so $(\bar{P} \rightarrow \bar{B})$,

(iv) Either $B M I$ indicates presence of Fetal Malnutrition or $P I$ asserts its absence (or both) $(1 \rightarrow \bar{B} \vee P)$.

Theuns (1989) restricts the number of implicational forms to $\left(2^{n}-2\right)$ where $n \geq 2$, by omitting the implication in which the antecedent is 1 and the implication in which the conclusion is 0 . These two implications can be written with the implication operator $(\rightarrow)$ replaced by the equality sign $(=)$. Both do not help in the construction of an implicational graph among the pertinent variables.

In Table 4, we highlight in bold certain implicational forms that seem more appealing for human understanding and verbal rephrasing as they are simple ones that involve only non-complemented literals. Note that all implications reported in Table 3 involve only non-complemented literals. This is a manifestation of the fact that the four metrics $C, B, P$, and $M$ have pairwise positive associations, dependencies or correlations, as expected through appeal to intuition, and as demonstrated earlier by Rushdi (2017) via more elaborate standard statistical methods. The results of this section attest indirectly to the genuine and good quality of data collected in the study of Rushdi (2017).

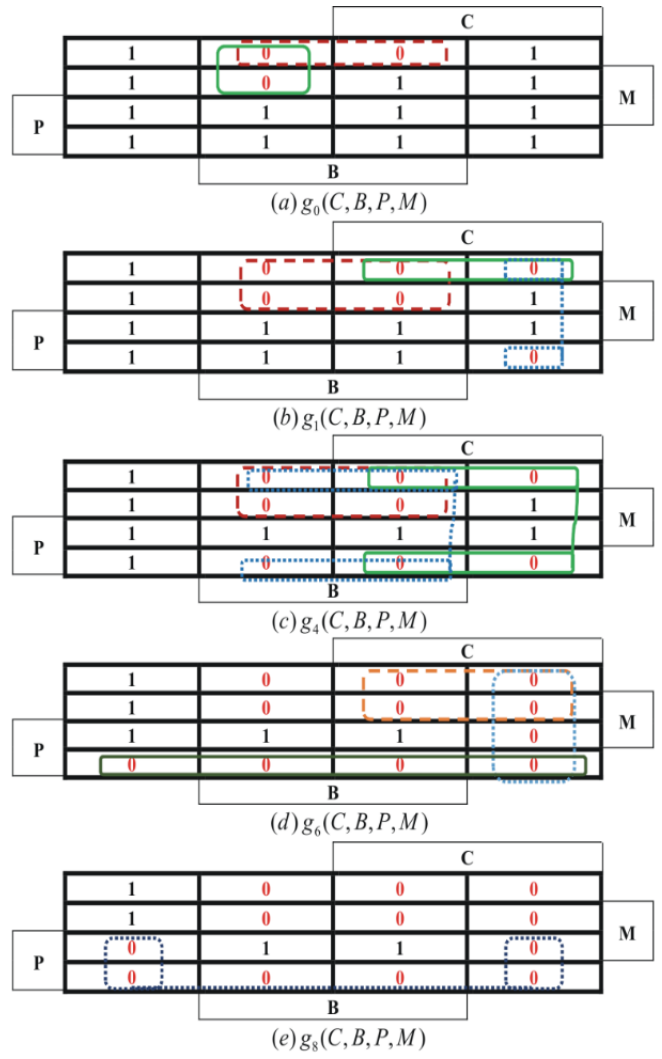

Fig. 8. Karnaugh-map representations for distinct Boolean functions obtained by dichotomization of the pseudo-Boolean function in Fig. 1 for various thresholds 
International Journal of Mathematical, Engineering and Management Sciences

Vol. 3, No. 3, 220-244, 2018

ISSN: 2455-7749

Table 3. Conclusions of Boolean Analysis for various plausible thresholds

\begin{tabular}{|c|c|c|}
\hline Threshold & Equational Conclusions & Implicational Conclusions \\
\hline \multirow{2}{*}{ 0 } & $B \bar{P} \bar{M}=0$ & $B \rightarrow P \vee M$ \\
\hline & $\bar{C} B \bar{P}=0$ & $B \rightarrow C \vee P$ \\
\hline \multirow{3}{*}{1} & $B \bar{P}=0$ & $B \rightarrow P$ \\
\hline & $C \bar{P} \bar{M}=0$ & $C \rightarrow P \vee M$ \\
\hline & $C \bar{B} \bar{M}=0$ & $C \rightarrow B \vee M$ \\
\hline \multirow{3}{*}{4} & $B \bar{P}=0$ & $B \rightarrow P$ \\
\hline & $C \bar{M}=0$ & $C \rightarrow M$ \\
\hline & $B \bar{M}=0$ & $B \rightarrow M$ \\
\hline \multirow{6}{*}{6} & $B \bar{P}=0$ & $B \rightarrow P$ \\
\hline & $C \bar{M}=0$ & $C \rightarrow M$ \\
\hline & $B \bar{M}=0$ & $B \rightarrow M$ \\
\hline & $C \bar{P}=0$ & $C \rightarrow P$ \\
\hline & $C \bar{B}=0$ & $C \rightarrow B$ \\
\hline & $P \bar{M}=0$ & $P \rightarrow M$ \\
\hline \multirow{7}{*}{8} & $B \bar{P}=0$ & $B \rightarrow P$ \\
\hline & $C \bar{M}=0$ & $C \rightarrow M$ \\
\hline & $B \bar{M}=0$ & $B \rightarrow M$ \\
\hline & $C \bar{P}=0$ & $C \rightarrow P$ \\
\hline & $C \bar{B}=0$ & $C \rightarrow B$ \\
\hline & $P \bar{M}=0$ & $P \rightarrow M$ \\
\hline & $P \bar{B}=0$ & $P \rightarrow B$ \\
\hline
\end{tabular}

Table 4. Various clausal (implicational) interpretations of deduced equations

\begin{tabular}{|c|c|c|c|c|}
\hline Equational Form & \multicolumn{4}{|c|}{ Clausal (Implicational) Forms } \\
\hline$B \bar{P}=0$ & $B \bar{P} \rightarrow 0$ & $\boldsymbol{B} \rightarrow \boldsymbol{P}$ & $\bar{P} \rightarrow \bar{B}$ & $1 \rightarrow \bar{B} \vee P$ \\
\hline \multirow{2}{*}{$B \bar{P} \bar{M}=0$} & $B \bar{P} \bar{M} \rightarrow 0$ & $\bar{P} \bar{M} \rightarrow \bar{B}$ & $B \bar{M} \rightarrow P$ & $B \bar{P} \rightarrow M$ \\
\cline { 2 - 5 } & $\bar{M} \rightarrow \bar{B} \vee P$ & $\bar{P} \rightarrow \bar{B} \vee M$ & $\boldsymbol{B} \rightarrow \boldsymbol{P} \vee \boldsymbol{M}$ & $1 \rightarrow \bar{B} \vee P \vee M$ \\
\hline
\end{tabular}

\section{Qualitative Comparative Analysis}

Qualitative Comparative Analysis ( $Q C A)$ was first introduced in the seminal paper of Ragin et al. (1984) and became well established via the celebrated text of Ragin (1989). In its first three decades, $Q C A$ bridged the gap between (and embodied some key strengths of) the qualitative and quantitative approaches typically used in humanities and social and political sciences (Rihoux, 2003; Marx et al., 2014). It has now permeated new areas (other than its conventional domains) such as engineering and medicine (Ragin, 1999; Bradley et al., 2007; Schensul et al., 2010; Jordan et al., 2011; Baumgartner and Thiem, 2017). In $Q C A$, a certain outcome is studied as a function of certain inputs, while in classical Boolean Analysis (see Sec. 4) a relation is studied between several variables that stand on equal footing, i.e., without presuming which are inputs and which are outputs. To apply $Q C A$ to the current problem of this paper, we must provide (even approximately) a perfect metric or gold standard that should serve as the outcome. From the statistical point of view, this standard should (in the ideal case) be constructed independently of all inputs, but we need, out of necessity, to relax this requirement herein. We employ for an approximate standard herein the previously-defined overall real voting function $F$ given by $F=0$ when $f<1$ and $F=1$ when $f \geq 1$, and $f$ is the average of the weighted raw metrics given by 
International Journal of Mathematical, Engineering and Management Sciences

Vol. 3, No. 3, 220-244, 2018

ISSN: 2455-7749

Eq. (5). We reiterate that $F$ is a Boolean indicator of the absence of the condition of Fetal Malnutrition, while its complement $\bar{F}$ is an indicator for the presence of that condition.

The common $Q C A$ practice is to use Boolean minimization to obtain the minimal sum of the pertinent output function $F$. This practice is definitely useful since it provides a compact characterization of the function. The minimal sum covers the asserted part of the function with as few as possible prime implicants. Unless the function considered is unate, the minimal sum typically does not contain all prime implicants. The disjunction of all prime implicants of $F$ is the complete sum or the Blake Canonical Form introduced earlier in Sec. 6, and as its names indicate it is canonical and complete (and hence not necessarily compact). Rushdi (2018) criticized mainstream $Q C A$ for not reporting the complete sum explicitly along with the minimal sum.

Since $Q C A$ is a technique based on Boolean algebra that typically involves only a few variables, it can conveniently be implemented via the Karnaugh map (Rushdi and Badawi, 2017a; Rushdi; 2018). This section utilizes the Karnaugh map as a tool of $Q C A$ for obtaining the Boolean indicators $F$ or $\bar{F}$ in terms of the four measures $(C, B, P$, and $M)$ used herein. An intuitionistic conjecture (based on concepts of Boolean voting) is that $F$ should be within the interval of functions bounded by two specific symmetric Boolean functions, namely, the 3-out-of-4 function $F_{3 / 4}$ and the 2-out-of-4 function $F_{2 / 4}$ (Rushdi and Rushdi, 2017). This conjecture is mathematically stated by the equations

$F_{3 / 4} \leq F \leq F_{2 / 4}$

$F_{3 / 4}=C B P \vee C B M \vee C P M \vee B P M$

$F_{2 / 4}=C B \vee C P \vee C M \vee B P \vee B M \vee P M$

A more concrete desirable feature of $F=F(C, B, P, M)$ is that it should be a coherent function, i.e., one satisfying the properties (Rushdi and Alturki, 2015):

1. Causality: The Karnaugh map is entered by 0 in the all-0 cell (the $\bar{C} \bar{B} \bar{P} \bar{M}$ cell, which is within the non-asserted domain of every variable), and entered by 1 in the all-1 cell (the CBPM cell, which is within the asserted domain of every variable).

2. Monotonicity: The map entry cannot decrease (from 1 to 0 ) upon transition from the nonasserted half-map domain of a variable to its asserted half-map domain, with states of other variables unchanged.

3. Relevancy: For every variable $i, 1 \leq i \leq n$, there exists at least one instance in which crossing the border of variable $i$ from its non-asserted half-map domain to its asserted half-map domain leads to an increase of cell entry (from 0 to 1 ).

Such a coherent function $F(C, B, P, M)$ is a unate function, and can have a sum-of-products (sop) representation consisting solely of uncomplemented literals. Examples of such a representation are the minimal sum of the function and its complete sum, which are the same in this case. Since the complete sum of any Boolean function is unique and canonical, this means that the minimal sum of a coherent $F(C, B, P, M)$ is also unique and canonical. 
International Journal of Mathematical, Engineering and Management Sciences

Vol. 3, No. 3, 220-244, 2018

ISSN: 2455-7749

We now reproduce results from Rushdi (2017) in the Karnaugh-map of Fig. 9, whose entries are values of the Boolean function $F(C, B, P, M)$, with the number of supporting cases of each entry included parenthetically. Clearly, a parenthetic number (or the sum of two parenthetic numbers) is the same as a cell entry in Fig. 1. Fig. 9 indicates that configurations corresponding to three map cells are never observed, while five other cells exhibit contradictory values (a " 0 " outcome for some observed cases and a " 1 " outcome for other observed cases). We assign don't-care values (d) to the non-observed configurations, and temporarily assign values of contradiction (C) in Fig. 10 to the contradictory cells.

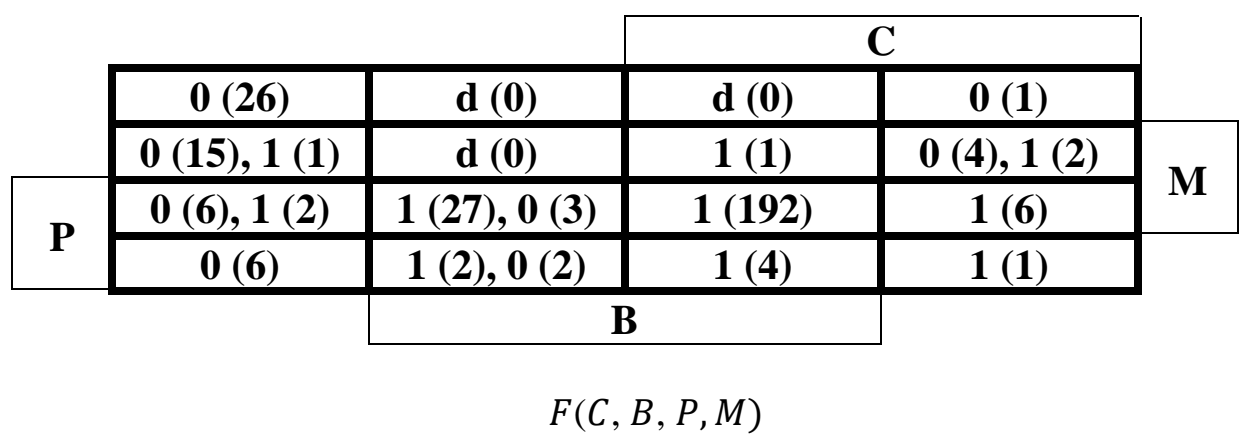

Fig. 9. Initial Karnaugh-map representation of the Boolean function $F(C, B, P, M)$ representing an approximate gold standard with supporting cases included parenthetically

Rihoux and de Meur (2009) explain why don't cares (Logical Remainders in their terminology) are useful and how they can be utilized in obtaining more parsimonious minimal formulas. However, they are concerned about making assumptions about non-observed cases. Rushdi and Badawi (2017a) note that if one refrain from utilizing don't-cares for minimization or for any purpose whatsoever, one is still setting certain unknown values to zero, and hence is still making unwarranted or unjustified assumptions about non-observed data. Crama et al. (1988) demand the availability of complete observations if a method is required to provide definite answers. In the present case, the debate about doesn't care is out of the question, since the current FM study is a closed one in the sense that the observed data is final rather than temporary or tentative.

The "contradiction" values (C) in Fig. 10 pose logical contradictions or inconsistencies that must be resolved before further processing. Techniques for resolving contradictions are discussed by Rihoux and de Meur (2009) and Jordan et al. (2011). Using the number of supporting cases as a resolving factor, we replace each of the contradictions $C_{1}, C_{2}$, and $C_{5}$ by 0 , and replace the contradiction $C_{3}$ by 1 . We cannot resolve the contradiction $C_{4}$, since it involves a 2-2 tie, and hence, we consider its two possibilities of 0 and 1 in Figs. 11(a) and 11(b), respectively. The corresponding final solutions are

$F_{1}=B \vee C P$

$F_{2}=C B \vee C P \vee B M$

We prefer the latter solution $F_{2}$ to the former one $F_{1}$ for several reasons. The solution $F_{1}$ is a noncoherent function in which the variable $M$ is a dummy irrelevant variable, and for which the 
International Journal of Mathematical, Engineering and Management Sciences

Vol. 3, No. 3, 220-244, 2018

ISSN: 2455-7749

anticipated guess (10)-(12) is violated, while the solution $F_{1}$ is a coherent function in which all variables are relevant, and which fits into the Boolean-predicted interval specified by equations (10)(12). The Banzhaf voting powers (Alturki and Rushdi, 2016) for the four variables $C, B, P$, and $M$ are 2, 6, 2, and 0 for the first solution and 4, 4, 2, and 2 for the second solution. The first solution assigns an importance to $B$ that is three times what it assigns to either $C$ or $P$. By contrast, the second solution considers $C$ and $B$ equally important and also treats $P$ and $M$ also as equally important, with the importance of a member of the first pair of metrics being double that of a member of the second pair. In her review of the extensive literature on Fetal Malnutrition, Rushdi (2017) effectively asserts that none of the four metrics $C, B, P$, and $M$ should be dispensed with, and that the metrics $C$ and $B$ are the most prominent of the four metrics. In fact, Rushdi (2017) notes that either one of the two metrics $C$ or $B$ is usually utilized as a standard metric in FM studies. Therefore, the second solution above fits well with the expert opinion prevailing in medical circles. This observation again indirectly attests to the genuine and good quality of data collected in the study of Rushdi (2017).

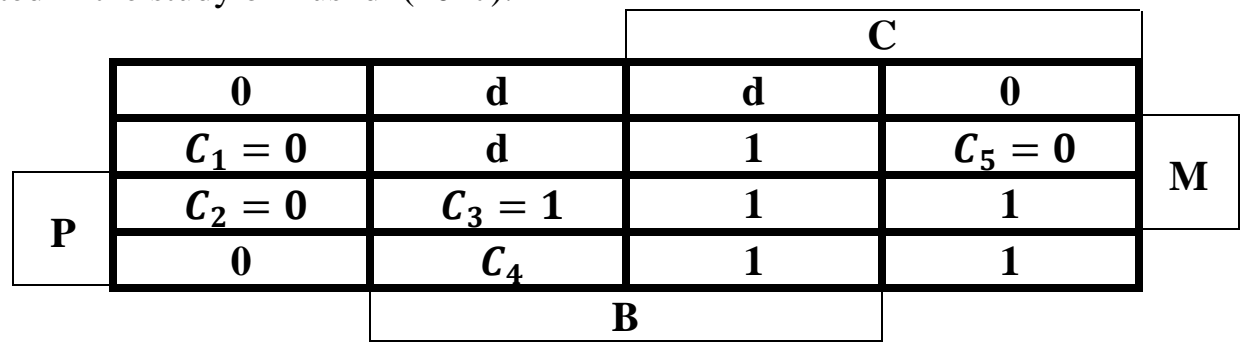

$F(C, B, P, M)$

Fig. 10. Final Karnaugh-map representation of the Boolean function $F(C, B, P, M)$ representing an approximate gold standard with three don't cares and a single unresolved contradiction

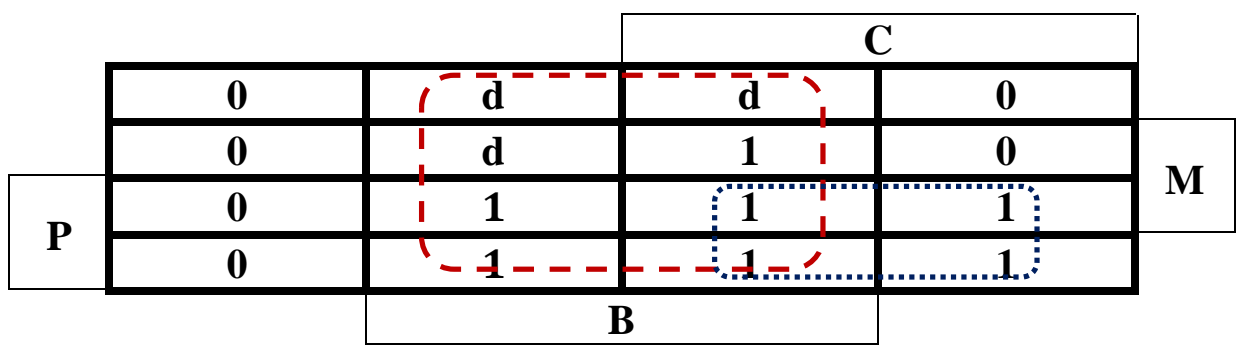

(a) $F_{1}(C, B, P, M)=B \vee C P$

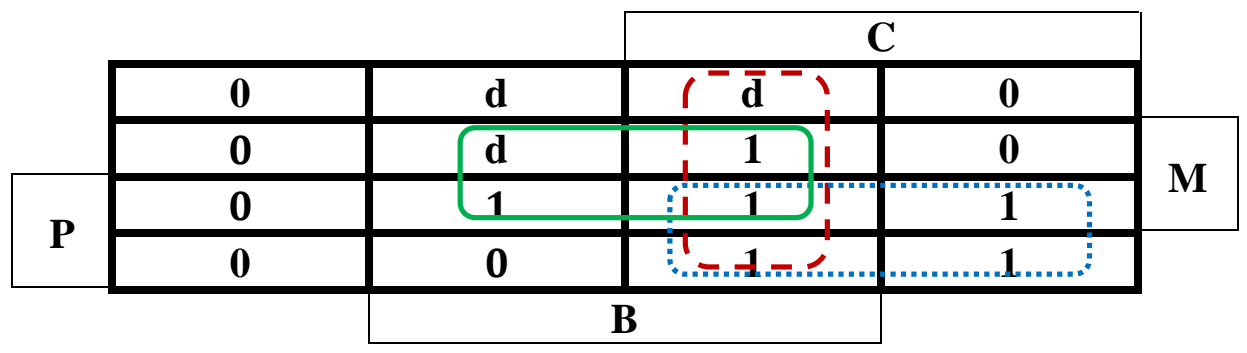

(b) $F_{2}(C, B, P, M)=C B \vee C P \vee B M$

Fig. 11. Two possible solutions depending on two arbitrary assignments for the unresolved contradiction 
International Journal of Mathematical, Engineering and Management Sciences

Vol. 3, No. 3, 220-244, 2018

ISSN: 2455-7749

\section{Conclusions}

We demonstrate the utility of the Karnaugh-map, as a pictorial manual tool of Boolean algebra, in the exploration of medical problems as exemplified herein by the problem of Fetal Malnutrition. The Karnaugh map serves as a convenient way for aggregating the set of clinical data available into a pseudo-Boolean function. The map is used to produce a two-by-two contingency matrix that relates an assessed test or metric to a reference or standard one. Each of these two metrics can be any of the map variables or a function of some or all of these variables, such as a voting function of all variables except one. While the map serves in this capacity as a supplement to statistical methods, it is also useful for certain non-statistical methods (specifically Boolean ones). The paper shows how the map entries are dichotomized via an appropriate threshold for use in Boolean Analysis (BA). The map also implements Qualitative Comparative Analysis ( $Q C A)$ for the given clinical data. The map variable-handling capability does not pose as a shortcoming in this case, since the number of variables involved (not only herein but in other typical medical problems as well) is typically very small. Both results of $B A$ and $Q C A$ attest indirectly to the genuine and good quality of data collected in the study of Rushdi (2017). The concepts and methods introduced herein are demonstrated through application to the aforementioned set of clinical data for the $F M$ problem, and can be extended to a wide variety of medical problems.

Boolean Analysis $(B A)$ and Qualitative Comparative Analysis ( $Q C A)$ are commonly believed to be variations of the same kind of analysis (Rushdi and Badawi, 2017a; 2017b). An offshoot contribution of this paper is to apply both analyses to the same case study, thereby revealing in a concrete manner some of the subtle differences between them. The $Q C A$ implemented herein via the Karnaugh map deals with a single distinguished effect (map output) expressed in terms of several inputs (map variables, presumably independent). Boolean Analysis is somewhat similarly implemented via a Karnaugh map, but now the map represents a function relating all pertinent (and maybe non-pertinent) variables. The map variables initially stand on equal footing, as the researcher cannot distinguish some of them as inputs and others as outputs. Therefore, Boolean Analysis neither needs a gold standard from the outset nor provides explicit information relating such a standard to the available metrics. It simply discovers possible interrelations among the available metrics, without relating them to the true metric or even attempting to identify the true metric. By contrast, $Q C A$ cannot be conducted in the absence of a gold standard or at least a good approximation thereof. The job of $Q C A$ is to express the (possibly approximate) gold standard in terms of the available metrics. Both $Q C A$ and $B A$ use curtailed information or dichotomized data, and hence are less demanding, from both the computational and pedagogical points of view. Their results are qualitative and might be viewed as secondary ones when compared to the primary statistical results. The $Q C A$ and $B A$ results are beneficial at least in alleviating the widespread feeling that statistics is the only game in town.

The method of $Q C A$ was used herein to explore possible relations among frequently used measures for Fetal Malnutrition. It can be used also to assess the determinants of this condition and its ramifications, thereby somewhat formalizing earlier qualitative investigations by Kramer (1987) and Kramer et al. (1990). The Karnaugh map used herein is a conventional one, which is capable of handling up to six dichotomous variables. Other potentially-useful variants of the map are the Multi-Valued Karnaugh Map (MVKM), which is capable of handling multi-valued variables, and the Variable-Entered Karnaugh Map (VEKM), which is capable of handling up to twelve variables (Rushdi, 2018). 
International Journal of Mathematical, Engineering and Management Sciences

Vol. 3, No. 3, 220-244, 2018

ISSN: 2455-7749

\section{Acknowledgements}

The authors are greatly indebted to Engineer Mostafa Ali Rushdi (lecturer at the Future University of Egypt) for the technical help he generously and proficiently offered during the preparation of this manuscript. They are really appreciative of his perseverance and his expertise.

\section{Appendix A: Prominent Measures or Indicators in the Statistical Analysis of Screening and Diagnostic Tests}

The medical literature abounds with measures or indicators for the statistical analysis of screening and diagnostic (confirmatory) tests, which are conducted on asymptomatic subjects and on subjects having symptoms (signs), respectively. There are many informative tutorials on the topic, including the contributions of Alonzo and Pepe (1999), Glas et al. (2003), Hawkins (2005), Fawcett (2006), Zhou et al. (2009), Powers (2011), Lewis and Torgerson (2012), Broemeling (2011), Leeflang (2014), Chughtai et al. (2015), Kent and Hancock (2016), Baveja and Aggarwal (2017), Porebski and Straszecka, (2018). Fig. A1 demonstrates the starting point of this analysis, which is a two-by-two contingency matrix (frequency or confusion matrix) for test or metric $i$ with respect to test or metric $j$, which can be viewed as a two-variable Karnaugh map representing a pseudo-Boolean function. As pointed out earlier, two dichotomous variables are involved, which are represented by the set $\{+1,-1\}$ of indices for the contingency matrix, or equivalently by the set $\{0,1\}$ for the Boolean variables of the Karnaugh map. The test or metric $i$ (typically a new test to be assessed) is reporting positive cases, in which the (usually adverse) condition is present, or reporting negative cases, in which this condition is absent. This test or metric is judged or evaluated by a reference or standard metric $j$ (typically a gold standard or the best test available), which has its own labeling of the cases, again as positives or negatives. If the reference metric $j$ agrees with the assessed metric $i$, then $j$ designates the cases of $i$ as "true", and if $j$ disagrees with $i$ then the reference metric designates the cases of the assessed one as "false".

The relation between the categorical dichotomous variables $i$ and $j$ is called an association or a correlation (albeit the latter term is better reserved for quantitative variables). Since each of these two variables has an inherent order, it is possible to label an association between them as positive or negative (Anderson and Finn, 1996). Such an association does not necessarily imply causality. In our present work, neither metric is expected to cause the other, but they are rather expected to be associated with a 'common cause.' The pile up of individual cases at the opposite corners of true positives and true negatives of the contingency table indicates a positive association between the two matrix variables. In the limiting case when cases at the other opposite corners (of false positives and false negatives) totally diminish, this association becomes a perfect one.

The four elements $T P_{i j}, F P_{i j}, F N_{i j}$, and $T N_{i j}$ in the two-by-two contingency matrix of Fig. A1 are called natural frequencies. They are simple non-negative integers that are not normalized and correspond to mutually exclusive conjunctive events. They simply count how many cases of the total sample size $N$ there are in each specific subcategory, and hence they add up to that total size (Hoffrage et al., 2002; Hoffrage et al., 2005; Gigerenzer et al., 2008). Each of these elements can be expressed as the sample size $N$ multiplied by a joint probability, which is a probability of an intersection of events concerning the metrics $i$ and $j$, namely

$T P_{i j}=N * P((i=+1) \cap(j=+1))$ 
International Journal of Mathematical, Engineering and Management Sciences

Vol. 3, No. 3, 220-244, 2018

ISSN: $2455-7749$

$F P_{i j}=N * P((i=+1) \cap(j=-1))$

$F N_{i j}=N * P((i=-1) \cap(j=+1))$

$T N_{i j}=N * P((i=-1) \cap(j=-1))$

The row-wise addition of these elements yields values proportional to the two marginal complementary probabilities concerning the reference metric $j$ (prevalence and non-prevalence according to $j$ ), namely

$T P_{i j}+F N_{i j}=N * P(j=+1)$

$F P_{i j}+T N_{i j}=N * P(j=-1)$

Likewise, the column-wise addition of the elements yields values proportional to the two marginal complementary probabilities concerning the assessed metric $i$ (prevalence and non-prevalence according to $i$ ), namely

$T P_{i j}+F P_{i j}=N * P(i=+1)$

$F N_{i j}+T N_{i j}=N * P(i=-1)$

Table A1 lists some of the measures or indicators commonly used in diagnostic medicine, express each of them in terms of the elements of the contingency matrix, and interprets each of them in terms of probability or conditional probability. This interpretation suggests many useful applications of the given measures. For example, the following two equations are expressions of the Total Probability Theorem that can be cast into a Signal Flow Graph or Channel Diagram, similar to the ones used in the study of digital communications or DNA replication (Rushdi, 2010).

$$
\begin{aligned}
& P(i=+1)=\operatorname{Sens}_{i j} * P(j=+1)+\left(1-\operatorname{Spec}_{i j}\right) * P(j=-1) \\
& =\operatorname{Sens}_{i j} * \operatorname{PTP}_{i j}+\left(1-\operatorname{Spec}_{i j}\right) *\left(1-\operatorname{PTP}_{i j}\right) \\
& P(i=-1)=\left(1-\operatorname{Sens}_{i j}\right) * P(j=+1)+\operatorname{Spec}_{i j} * P(j=-1) \\
& =\left(1-\operatorname{Sens}_{i j}\right) * \operatorname{PTP}_{i j}+\operatorname{Spec}_{i j} *\left(1-\operatorname{PTP}_{i j}\right)
\end{aligned}
$$

An Inverse Channel Diagram might be constructed of the following two inverse equations

$$
\begin{aligned}
& P(j=+1)=P P V_{i j} * P(i=+1)+\left(1-N P V_{i j}\right) * P(i=-1) \\
& P(j=-1)=\left(1-P P V_{i j}\right) * P(i=+1)+N P V_{i j} * P(i=-1)
\end{aligned}
$$

Application of Bayes' Theorem (Hall, 1967; Winkler and Smith, 2004; Thomas et al., 2011; Shindo, et al., 2012) reduces Equations (A9) and (A10) to

$$
P P V_{i j}=\left(\text { Sens }_{i j} * P T P_{i j}\right) /\left(\text { Sens }_{i j} * P T P_{i j}+\left(1-\operatorname{Spec}_{i j}\right) *\left(1-P T P_{i j}\right)\right)
$$


International Journal of Mathematical, Engineering and Management Sciences

Vol. 3, No. 3, 220-244, 2018

ISSN: 2455-7749

$N P V_{i j}=\left(\operatorname{Spec}_{i j} *\left(1-P T P_{i j}\right)\right) /\left(\left(1-\operatorname{Sens}_{i j}\right) * P T P_{i j}+\operatorname{Spec}_{i j} *\left(1-P T P_{i j}\right)\right)$

\begin{tabular}{|c|c|c|}
\hline$i$ & +1 & -1 \\
\hline+1 & $\begin{array}{c}T P_{i j} \\
\text { (True Positives) }\end{array}$ & $\begin{array}{c}F P_{i j} \\
\text { (False Positives) }\end{array}$ \\
\hline-1 & $\begin{array}{c}F N_{i j} \\
\text { (False Negatives) }\end{array}$ & $\begin{array}{c}T N_{i j} \\
\text { (True Negatives) }\end{array}$ \\
\hline
\end{tabular}

(a)

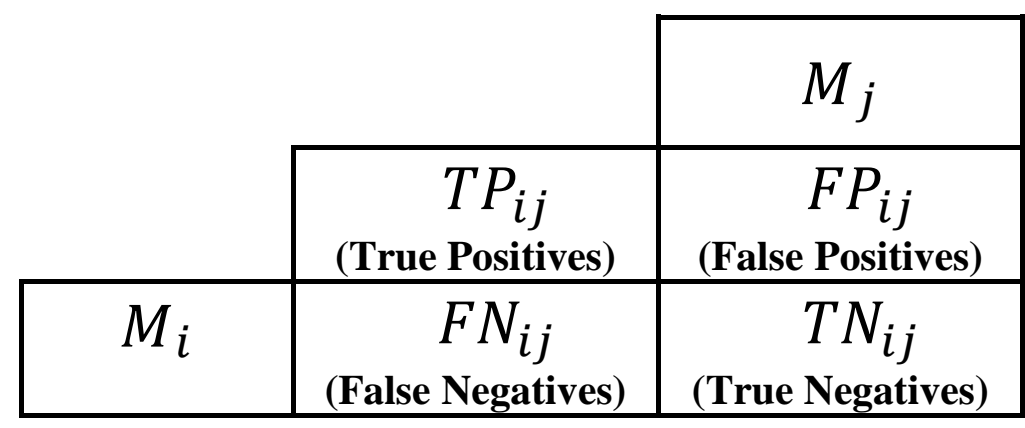

(b)

Fig. A1. The two-by-two Contingency Matrix (a) of test or metric $i$ with respect to test or metric $j$ viewed as a two-variable Karnaugh map (b) representing a pseudo-Boolean function

Most of the measures in Table A1 exist in pairs such as $\left\{\operatorname{Sens}_{i j}, \operatorname{Spec}_{i j}\right\}$ and $\left\{P P V_{i j}, N P V_{i j}\right\}$, but a few serve as global measures or single indicators, namely total error and accuracy, pre-test prevalence and odds, DOR, inverse DOR, and Index of Association. Each of the measures in Table A1 is a probability and hence belongs to the unit interval $[0.0,1.0]$, with the exception of Youden's Index (Informedness), Markedness and the Index of Association, which belong to $[-1.0,+1.0]$, and certain probability ratios (the DOR, its inverse, various likelihood ratios and test odds), which belong to $[0.0, \infty)$. A measure interpretable as a probability is either a ratio based on a column total, or a ratio based on a row total, or a ratio based on the grand total. Unlike other measures, the Index of Association does not depend on which metric or test is assessed relative to which $\left(\emptyset_{i j}=\emptyset_{j i}\right)$. Typically, the two metrics or tests are highly dependent. They are independent when the condition $\left\{T P_{i j} * T N_{i j}=F P_{i j} * F N_{i j}\right\}$ is satisfied (Tsumoto, 2009), which implies $\left\{\varnothing_{i j}=0\right\}$ and $\left\{D O R_{i j}=\right.$ 1\}. The interesting case of perfect positive association occurs when $\left\{F P_{i j}=F N_{i j}=0\right\}$ at which $\left\{\varnothing_{i j}=+1\right\}$ and $\left\{D O R_{i j} \rightarrow \infty\right\}$. 
International Journal of Mathematical, Engineering and Management Sciences

Vol. 3, No. 3, 220-244, 2018

ISSN: 2455-7749

Table A1. Commonly used measures or indicators in diagnostic medicine

\begin{tabular}{|c|c|c|}
\hline Measure or indicator & In terms of natural frequencies & $\begin{array}{c}\text { Interpretation as probability or } \\
\text { conditional probability }\end{array}$ \\
\hline $\begin{array}{l}\text { Sensitivity (True Positive Rate (TPR), } \\
\text { Recall, Probability of Detection) }\end{array}$ & Sens $_{i j}=T P_{i j} /\left(T P_{i j}+F N_{i j}\right)$ & Sens $_{i j}=P(i=+1 \mid j=+1)$ \\
\hline $\begin{array}{c}\text { Specificity } \\
\text { (True Negative Rate (TNR)) }\end{array}$ & $\operatorname{Spec}_{i j}=T N_{i j} /\left(T N_{i j}+F P_{i j}\right)$ & $\operatorname{Spec}_{i j}=P(i=-1 \mid j=-1)$ \\
\hline $\begin{array}{c}\text { Precision (Positive Predictive Value } \\
\text { (PPV)) }\end{array}$ & $P P V_{i j}=T P_{i j} /\left(T P_{i j}+F P_{i j}\right)$ & $P P V_{i j}=P(j=+1 \mid i=+1)$ \\
\hline Negative Predictive Value (NPV) & $N P V_{i j}=T N_{i j} /\left(T N_{i j}+F N_{i j}\right)$ & $N P V_{i j}=P(j=-1 \mid i=-1)$ \\
\hline False Negative Rate (FNR) & $F N R_{i j}=1-$ Sens $_{i j}=F N_{i j} /\left(T P_{i j}+F N_{i j}\right)$ & $F N R_{i j}=P(i=-1 \mid j=+1)$ \\
\hline $\begin{array}{c}\text { False Positive Rate (FPR) (Fall-Out, } \\
\text { False Alarm) }\end{array}$ & $F P R_{i j}=1-\operatorname{Spec}_{i j}=F P_{i j} /\left(T N_{i j}+F P_{i j}\right)$ & $F P R_{i j}=P(i=+1 \mid j=-1)$ \\
\hline False Discovery Rate (FDR) & $\begin{array}{l}F D R_{i j}=1-P P V_{i j}= \\
F P_{i j} /\left(T P_{i j}+F P_{i j}\right)\end{array}$ & $F D R_{i j}=P(j=-1 \mid i=+1)$ \\
\hline False Omission Rate (FOR) & $\begin{array}{l}F O R_{i j}=1-N P V_{i j}= \\
F N_{i j} /\left(T N_{i j}+F N_{i j}\right) \\
\end{array}$ & $F O R_{i j}=P(j=+1 \mid i=-1)$ \\
\hline Likelihood Ratio for Positive Test & $(L R+)_{i j}=\operatorname{Sens}_{i j} /\left(1-\operatorname{Spec}_{i j}\right)$ & $\begin{array}{c}(L R+)_{i j}=P(i=+1 \mid j=+1) / P(i= \\
+1 \mid j=-1)\end{array}$ \\
\hline Likelihood Ratio for Negative Test & $(L R-)_{i j}=\left(1-\right.$ Sens $\left._{i j}\right) /$ Spec $_{i j}$ & $\begin{array}{c}(L R-)_{i j}=P(i=-1 \mid j=+1) / P(i= \\
-1 \mid j=-1)\end{array}$ \\
\hline Diagnostic Odds Ratio & $D O R_{i j}=\left(T P_{i j} * T N_{i j}\right) /\left(F P_{i j} * F N_{i j}\right)$ & $D O R_{i j}=(L R+)_{i j} /(L R-)_{i j}$ \\
\hline Inverse of the DOR & $D O R_{i j}^{-1}=\left(F P_{i j} * F N_{i j}\right) /\left(T P_{i j} * T N_{i j}\right)$ & $D O R_{i j}^{-1}=(L R-)_{i j} /(L R+)_{i j}$ \\
\hline Youden's Index (Informedness) & $Y I_{i j}=\operatorname{Sens}_{i j}+\operatorname{Spec}_{i j}-1$ & $\begin{array}{c}Y I_{i j}=P(i=+1 \mid j=+1)+P(i= \\
-1 \mid j=-1)-1\end{array}$ \\
\hline Markedness & $M_{i j}=P P V_{i j}+N P V_{i j}-1$ & $\begin{array}{c}M_{i j}=P(j=+1 \mid i=+1)+P(j= \\
-1 \mid i=-1)-1\end{array}$ \\
\hline Error of the First Kind & $E 1_{i j}=F P_{i j} / N$ & $E 1_{i j}=P((i=+1) \cap(j=-1))$ \\
\hline Error of the Second Kind & $E 2_{i j}=F N_{i j} / N$ & $E 2_{i j}=P((i=-1) \cap(j=+1))$ \\
\hline Total Diagnostic Error & $E_{i j}=\left(F P_{i j}+F N_{i j}\right) / N$ & $E_{i j}=E 1_{i j}+E 2_{i j}=P(i \neq j)$ \\
\hline Diagnostic Accuracy & $A_{i j}=\left(T P_{i j}+T N_{i j}\right) / N$ & $A_{i j}=1-E_{i j}=P(i=j)$ \\
\hline Pre-Test Prevalence & $P T P_{i j}=\left(T P_{i j}+F N_{i j}\right) / N$ & $P T P_{i j}=P(j=+1)$ \\
\hline Pre-Test Odds & $P T O_{i j}=\left(T P_{i j}+F N_{i j}\right) /\left(F P_{i j}+T N_{i j}\right)$ & $P T O_{i j}=P(j=+1) / P(j=-1)$ \\
\hline Post-Positive-Test Odds & $P P T O_{i j}=P T O_{i j}(L R+)_{i j}=T P_{i j} / F P_{i j}$ & $\begin{array}{c}\text { PPTO }_{i j}=P((i=+1) \cap(j=+1)) \\
/ P((i=+1) \cap(j=-1))\end{array}$ \\
\hline Post-Negative-Test Odds & $P N T O_{i j}=P T O_{i j}(L R-)_{i j}=F N_{i j} / T N_{i j}$ & $\begin{array}{c}P^{P N T O_{i j}}=P((i=-1) \cap(j=+1)) \\
/ P((i=-1) \cap(j=-1))\end{array}$ \\
\hline $\begin{array}{l}\text { Index of Association or Matthews } \\
\text { Correlation Coefficient (MCC) } \\
\qquad \emptyset_{i j}=\emptyset_{j i}\end{array}$ & $\begin{array}{c}\emptyset_{i j}=\emptyset_{j i}=\left(T P_{i j} * T N_{i j}-F P_{i j} *\right. \\
\left.F N_{i j}\right) / S Q R T\left(\left(T P_{i j}+F N_{i j}\right)\left(T P_{i j}+\right.\right. \\
\left.\left.F P_{i j}\right)\left(T N_{i j}+F P_{i j}\right)\left(T N_{i j}+F N_{i j}\right)\right)\end{array}$ & $\begin{array}{c}\emptyset_{i j}=\emptyset_{j i}=\operatorname{SQRT}(P(i=+1 \mid j= \\
+1) P(i=-1 \mid j=-1) P(j=+1 \mid i= \\
+1) P(j=-1 \mid i=-1))-S Q R T(P(i= \\
-1 \mid j=+1) P(i=+1 \mid j=-1) P(j= \\
-1 \mid i=+1) P(j=+1 \mid i=-1))\end{array}$ \\
\hline
\end{tabular}


International Journal of Mathematical, Engineering and Management Sciences

Vol. 3, No. 3, 220-244, 2018

ISSN: 2455-7749

\section{References}

Alonzo, T. A., \& Pepe, M. S. (1999). Using a combination of reference tests to assess the accuracy of a new diagnostic test. Statistics in Medicine, 18(22), 2987-3003.

Alturki, A. M., \& Rushdi, A. M. A., (2016). Weighted voting systems: a threshold-Boolean perspective, Journal of Engineering Research, 4(1), 125-143.

Anderson, T. W., \& Finn, J. D. (1996). Summarizing Multivariate Data: Association between Categorical Variables, Chapter 6 in The New Statistical Analysis of Data. Springer Science \& Business Media, pp 177-230

Baumgartner, M. (2009). Uncovering deterministic causal structures: a Boolean approach. Synthese, 170(1), 71-96.

Baumgartner, M., \& Thiem, A. (2017). Often trusted but never (properly) tested: evaluating qualitative comparative analysis. Sociological Methods \& Research, Online first 3 May, 2017.

Baveja, C. P., \& Aggarwal, P. (2017). Statistical analysis of microbiological diagnostic tests. Indian Journal of Medical Microbiology, 35(2), 184-193.

Bhambu, L., \& Kumar, D. (2015). A novel approach for classification on breast cancer data set. International Journal of Advanced Research in Computer Science and Software Engineering, 5(7), 1118-1123.

Bradley, E. H., Curry, L. A., \& Devers, K. J. (2007). Qualitative data analysis for health services research: developing taxonomy, themes, and theory. Health Services Research, 42(4), 1758-1772.

Broemeling, L. D. (2011). Advanced Bayesian methods for medical test accuracy. CRC Press, Boca Raton, FL, USA.

Brown, F. M. (1990). Boolean reasoning: the logic of Boolean equations, Kluwer Academic Publishers, Boston, USA.

Chughtai, A., Kelly, A. M., \& Cronin, P. (2015). How to perform a critical appraisal of diagnostic tests: 7 steps. Pediatric Radiology, 45(6), 793-803.

Crama, Y., Hammer, P. L., \& Ibaraki, T. (1988). Cause-effect relationships and partially defined Boolean functions. Annals of Operations Research, 16(1), 299-325.

De Felice, C., Cortelazzo, A., Leoncini, S., Signorini, C., Hayek, J., \& Ciccoli, L. (2016). Statistics, biomedicine and scientific fraud. Journal of the Siena Academy of Sciences, Focus on Biostatistics, $7(1), 15-22$.

DeCoster, J., Iselin, A. M. R., \& Gallucci, M. (2009). A conceptual and empirical examination of justifications for dichotomization. Psychological Methods, 14(4), 349-366.

Degenne, A., \& Lebeaux, M. O. (1996). Boolean analysis of questionnaire data. Social Networks, 18(3), 231-245.

Dendukuri, N., Schiller, I., Joseph, L., \& Pai, M. (2012). Bayesian meta-analysis of the accuracy of a test for tuberculous pleuritis in the absence of a gold standard reference. Biometrics, 68(4), 1285-1293.

Fawcett, T. (2006). An introduction to ROC analysis. Pattern Recognition Letters, 27(8), 861-874.

Feinstein, A. R. (1963). Boolean algebra and clinical taxonomy: analytic synthesis of the general spectrum of a human disease. New England Journal of Medicine, 269(18), 929-938.

Flament, C. (1965). L'analyse Booléenne de questionnaires (Boolean analysis of questionnaires). Mathématiques et Sciences Humaines, 12, 3-10. 
International Journal of Mathematical, Engineering and Management Sciences

Vol. 3, No. 3, 220-244, 2018

ISSN: 2455-7749

Flament, C. (1976). L'analyse Booléenne de questionnaire (the Boolean analysis of a questionnaire), Mouton, Paris, France.

Gigerenzer, G., \& Marewski, J. N. (2015). Surrogate science: the idol of a universal method for scientific inference. Journal of Management, 41(2), 421-440.

Gigerenzer, G., Gaissmaier, W., Kurz-Milcke, E., Schwartz, L. M., \& Woloshin, S. (2008). Helping doctors and patients make sense of health statistics. Psychological Science in the Public Interest, 8(2), 53-96.

Glantz, S. A. (1980). Biostatistics: how to detect, correct and prevent errors in the medical literature. Circulation, 61(1), 1-7.

Glas, A. S., Lijmer, J. G., Prins, M. H., Bonsel, G. J., \& Bossuyt, P. M. (2003). The diagnostic odds ratio: a single indicator of test performance. Journal of Clinical Epidemiology, 56(11), 1129-1135.

Hall, G. H. (1967). The clinical application of Bayes' theorem. The Lancet, 290(7515), 555-557.

Hammer, P. L., \& Bonates, T. O. (2006). Logical analysis of data-an overview: from combinatorial optimization to medical applications. Annals of Operations Research, 148(1), 203-225.

Hawkins, R. C. (2005). The evidence based medicine approach to diagnostic testing: practicalities and limitations. Clinical Biochemist Reviews, 26(2), 7-18.

Hoffrage, U., Gigerenzer, G., Krauss, S., \& Martignon, L. (2002). Representation facilitates reasoning: what natural frequencies are and what they are not. Cognition, 84(3), 343-352.

Hoffrage, U., Kurzenhäuser, S., \& Gigerenzer, G. (2005). Understanding the results of medical tests: why the representation of statistical information matters. In Bibace, R., et al. (Editors), Science and Medicine in Dialogue: Thinking Through particulars and Universals, 83-98.

Jordan, E., Gross, M. E., Javernick-Will, A. N., \& Garvin, M. J. (2011). Use and misuse of Qualitative Comparative Analysis. Construction Management and Economics, 29(11), 1159-1173.

Joseph, L., Gyorkos, T. W., \& Coupal, L. (1995). Bayesian estimation of disease prevalence and the parameters of diagnostic tests in the absence of a gold standard. American Journal of Epidemiology, 141(3), 263-272.

Kent, P., \& Hancock, M. J. (2016). Interpretation of dichotomous outcomes: sensitivity, specificity, likelihood ratios, and pre-test and post-test probability. Journal of Physiotherapy, 62(4), 231-233.

Kramer, M. S. (1987). Determinants of low birth weight: methodological assessment and meta-analysis. Bulletin of the World Health Organization, 65(5), 663-737.

Kramer, M. S., Olivier, M., McLean, F. H., Dougherty, G. E., Willis, D. M., \& Usher, R. H. (1990). Determinants of fetal growth and body proportionality. Pediatrics, 86(1), 18-26.

Lang, T. (2004). Twenty statistical errors even you can find in biomedical research articles. Croatian Medical Journal, 45(4), 361-370.

Leeflang, M. M. G. (2014). Systematic reviews and meta analyses of diagnostic test accuracy. Clinical Microbiology and Infection, 20(2), 105-113.

Lewis, F. I., \& Torgerson, P. R. (2012). A tutorial in estimating the prevalence of disease in humans and animals in the absence of a gold standard diagnostic. Emerging Themes in Epidemiology, 9, 1-8.

Lin, P. C. K., \& Khatri, S. P. (2014). Logic synthesis for genetic diseases: modeling disease behavior using Boolean networks. Springer Science \& Business Media, New York, NY, USA.

Lusted, L. B., \& Ledley, R. S. (1960). Mathematical models in medical diagnosis. Academic Medicine, 35(3), 214-222. 
International Journal of Mathematical, Engineering and Management Sciences

Vol. 3, No. 3, 220-244, 2018

ISSN: 2455-7749

Marshall, R. J. (1986). Partitioning methods for classification and decision making in medicine. Statistics in Medicine, 5(5), 517-526.

Marshall, R. J. (2001). The use of classification and regression trees in clinical epidemiology. Journal of Clinical Epidemiology, 54(6), 603-609.

Marx, A., Rihoux, B., \& Ragin, C. (2014). The origins, development, and application of qualitative comparative analysis: the first 25 years. European Political Science Review, 6(1), 115-142.

O’Neill, D. (2015). Measuring obesity in the absence of a gold standard. Economics \& Human Biology, 17, 116-128.

Ogihara, H., Fujita, Y., Hamamoto, Y., Iizuka, N., \& Oka, M. (2013, November). Classification based on Boolean algebra and its application to the prediction of recurrence of liver cancer. In IEEE $20132^{\text {nd }}$ IAPR Asian Conference on Pattern Recognition (ACPR), pp. 838-841.

Parikh, R., Mathai, A., Parikh, S., Sekhar, G. C., \& Thomas, R. (2008). Understanding and using sensitivity, specificity and predictive values. Indian Journal of Ophthalmology, 56(1), 45-50.

Porebski, S., \& Straszecka, E. (2018). Extracting easily interpreted diagnostic rules. Information Sciences, 426, 19-37.

Powers, D. M. (2011). Evaluation: from precision, recall and F-measure to ROC, informedness, markedness and correlation. Journal of Machine Learning Technologies. 2(1), 37-63.

Ragin, C. C. (1999). Using qualitative comparative analysis to study causal complexity. Health Services Research, 34(5 Pt 2), 1225-1239.

Ragin, C. C. (1989). The comparative method: moving beyond qualitative and quantitative strategies, Berkeley, Los Angeles and London, University of California Press.

Ragin, C. C., Mayer, S. E., \& Drass, K. A. (1984). Assessing discrimination: a Boolean approach. American Sociological Review, 49(2), 221-234.

Reitsma, J. B., Rutjes, A. W., Khan, K. S., Coomarasamy, A., \& Bossuyt, P. M. (2009). A review of solutions for diagnostic accuracy studies with an imperfect or missing reference standard. Journal of Clinical Epidemiology, 62(8), 797-806.

Rihoux, B. (2003). Bridging the gap between the qualitative and quantitative worlds? a retrospective and prospective view on qualitative comparative analysis. Field Methods, 15(4), 351-365.

Rihoux, B., \& de Meur, G. (2009). Crisp-set qualitative comparative analysis (csQCA), In B. Rihoux, B. and Ragin, C. C. (Editors), Configurational Comparative Methods: Qualitative Comparative Analysis (QCA) and Related Techniques, Thousand Oaks, CA, Sage, pp. 33-69.

Rindskopf, D., \& Rindskopf, W. (1986). The value of latent class analysis in medical diagnosis. Statistics in Medicine, 5(1), 21-27.

Royston, P., Altman, D. G., \& Sauerbrei, W. (2006). Dichotomizing continuous predictors in multiple regression: a bad idea. Statistics in Medicine, 25(1), 127-141.

Rushdi, A. A. (2010). A mathematical model of DNA replication. International Magazine on Advances in Computer Science and Telecommunications (IMACST), 1(1), 23-30.

Rushdi, A. M., \& Rushdi M. A. (2017). Switching-algebraic analysis of system reliability, Chapter 6 in Ram, M. and Davim, P. (Editors). Advances in Reliability and System Engineering. Management and Industrial Engineering Series. Springer International Publishing, Cham, Switzerland, pp.139-161.

Rushdi, A. M., \& Rushdi, M. A. (2018). Mathematics and examples of the modern syllogistic method of propositional logic, In Ram, M. (Editor), Mathematics Applied in Information Systems, Bentham Science Publishers, Emirate of Sharjah, United Arab Emirates. 
International Journal of Mathematical, Engineering and Management Sciences

Vol. 3, No. 3, 220-244, 2018

ISSN: 2455-7749

Rushdi, A. M. (1986). Map differentiation of switching functions. Microelectronics and Reliability, 26(5), 891-907.

Rushdi, A. M. A., \& Alturki, A. M. (2015). Reliability of coherent threshold systems. Journal of Applied Sciences, 15(3), 431-443.

Rushdi, A. M. A., \& Badawi, R. M. S. (2017a). Karnaugh-map utilization in Boolean analysis: The case of war termination. Journal of Qassim University: Engineering and Computer Sciences, 10(1), 53-88.

Rushdi, A. M. A., \& Badawi, R. M. S. (2017b). Karnaugh map utilization in coincidence analysis, Journal of King Abdulaziz University: Faculty of Computers and Information Technology, 6(1), in press.

Rushdi, A. M. A. (2018). Utilization of Karnaugh maps in multi-value qualitative comparative analysis, International Journal of Mathematical, Engineering and Management Sciences, 3(1), 28-46.

Rushdi, A. M., \& Ba-Rukab, O. M. (2017). Map calculation of the Shapley-Shubik voting powers: an example of the European Economic Community. International Journal of Mathematical, Engineering and Management Sciences, 2(1), 17-29.

Rushdi, R. A. (2017). Fetal Malnutrition: Assessment by the CANS score versus Anthropometry and Impact on Early Neonatal Morbidities, Unpublished Master Thesis, Department of Pediatrics, Kasr Al-Ainy School of Medicine, Cairo University, Cairo, Egypt, Available online at https://www.researchgate.net/profile/Rufaidah_Rushdi/contributions.

Schensul, J. J., Chandran, D., Singh, S. K., Berg, M., Singh, S., \& Gupta, K. (2010). The use of qualitative comparative analysis for critical event research in alcohol and HIV in Mumbai, India. AIDS and Behavior, 14(1), 113-125.

Shindo, T., Takahashi, T., Okamoto, T., \& Kuraishi, T. (2012). Evaluation of diagnostic results by Bayes' theorem. IEEJ Transactions on Electrical and Electronic Engineering, 7(5), 450-453.

Strasak, A. M., Zaman, Q., Marinell, G., Pfeiffer, K. P., \& Ulmer, H. (2007). The use of statistics in medical research: a comparison of The New England Journal of Medicine and Nature Medicine. The American Statistician, 61(1), 47-55.

Theuns, P. (1989). Predicting an optimal threshold in Boolean analysis of questionnaires. In Roskam, E. E. (Editor), Mathematical Psychology in Progress, Springer-Verlag Berlin Heidelberg, 329-343.

Theuns, P. (1994). A dichotomization method for Boolean analysis of quantifiable co-occurrence data. In G. Fischer and D. Laming (Editors), Contributions to Mathematical Psychology, Psychometrics, and Methodology, 2nd Ed., pp. 389-402, New York, USA, Springer.

Theuns, P. (1999). A Boolean approach to hierarchical data analysis: an overview. In $30^{\text {th }}$ Meeting of the European Mathematical Psychology Group, Mannheim, Germany, 1-18.

Thomas, R., Mengersen, K., Parikh, R. S., Walland, M. J., \& Muliyil, J. (2011). Enter the reverend: introduction to and application of Bayes' theorem in clinical ophthalmology. Clinical \& Experimental Ophthalmology, 39(9), 865-870.

Tsumoto, S. (2009). Contingency matrix theory: statistical dependence in a contingency table. Information Sciences, 179(11), 1615-1627.

Van Loo, H. M., \& Romeijn, J. W. (2015). Psychiatric comorbidity: fact or artifact? Theoretical Medicine and Bioethics, 36(1), 41-60.

Winkler, R. L., \& Smith, J. E. (2004). On uncertainty in medical testing. Medical Decision Making, 24(6), 654-658.

Zhou, X. H., McClish, D. K., \& Obuchowski, N. A. (2009). Statistical methods in diagnostic medicine (Vol. 569). John Wiley \& Sons, New York, NY, USA. 